Portland State University

PDXScholar

$10-2016$

\title{
Puddle Jumping: Spontaneous Ejection of Large Liquid Droplets from Hydrophobic Surfaces During Drop Tower Tests
}

\author{
Babek Attari \\ Portland State University \\ Mark M. Weislogel \\ Portland State University, weisloge@pdx.edu \\ Andrew Paul Wollman \\ Portland State University, drew.wollman@gmail.com \\ Yongkang Chen \\ Portland State University \\ Trevor Snyder \\ 3D Systems Corporation
}

Follow this and additional works at: https://pdxscholar.library.pdx.edu/mengin_fac

Part of the Fluid Dynamics Commons, and the Materials Science and Engineering Commons Let us know how access to this document benefits you.

\section{Citation Details}

Attari, B., Weislogel, M., Wollman, A., Chen, Y., \& Snyder, T. (2016). Puddle jumping: Spontaneous ejection of large liquid droplets from hydrophobic surfaces during drop tower tests. Physics of Fluids, 28(10), 102104.

This Article is brought to you for free and open access. It has been accepted for inclusion in Mechanical and Materials Engineering Faculty Publications and Presentations by an authorized administrator of PDXScholar. Please contact us if we can make this document more accessible: pdxscholar@pdx.edu. 


\section{Puddle jumping: Spontaneous ejection of large liquid droplets from hydrophobic surfaces during drop tower tests}

B. Attari, M. Weislogel, A. Wollman, Y. Chen, and T. Snyder

Citation: Physics of Fluids 28, 102104 (2016); doi: 10.1063/1.4963686

View online: http://dx.doi.org/10.1063/1.4963686

View Table of Contents: http://aip.scitation.org/toc/phf/28/10

Published by the American Institute of Physics

\section{Articles you may be interested in}

Spontaneous rotation of an ice disk while melting on a solid plate

Physics of Fluids 28, 123601123601 (2016); 10.1063/1.4967399

Numerical study on the freely falling plate: Effects of density ratio and thickness-to-length ratio

Physics of Fluids 28, 103603103603 (2016); 10.1063/1.4963242

Drop impact on a fiber

Physics of Fluids 28, 042001042001 (2016); 10.1063/1.4945103

Finding order in complexity: A study of the fluid dynamics in a three-dimensional branching network

Physics of Fluids 28, 123602123602 (2016); 10.1063/1.4971315

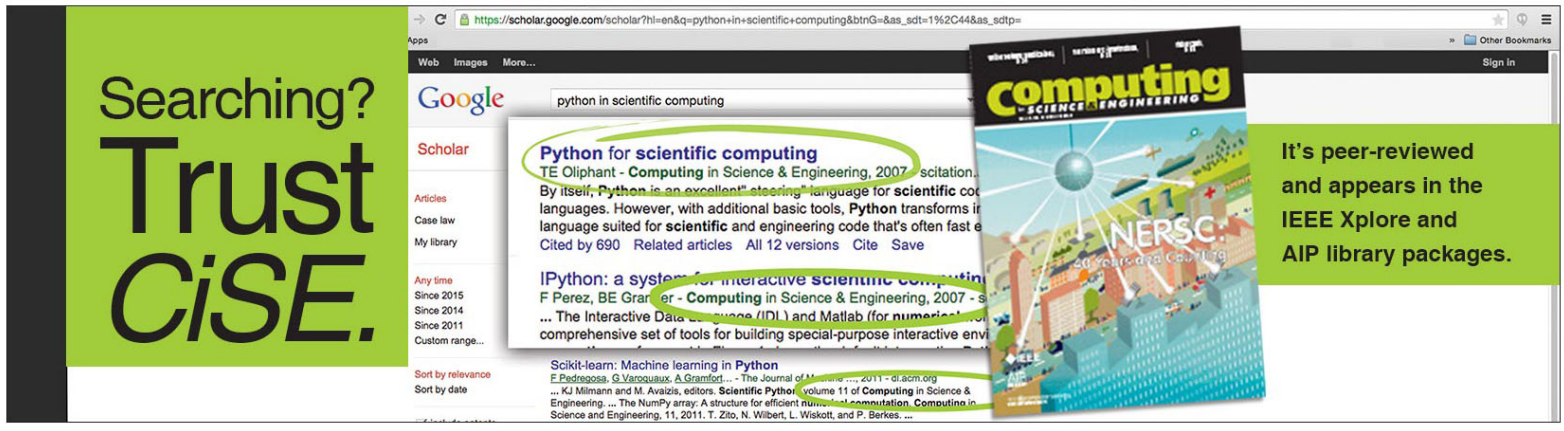




\title{
Puddle jumping: Spontaneous ejection of large liquid droplets from hydrophobic surfaces during drop tower tests
}

\author{
B. Attari, ${ }^{1}$ M. Weislogel, ${ }^{1, \text { a) }}$ A. Wollman, ${ }^{1}$ Y. Chen, ${ }^{1}$ and T. Snyder ${ }^{2}$ \\ ${ }^{1}$ Portland State University, Portland, Oregon 97201, USA \\ 23-D Systems, Wilsonville, Oregon 97070, USA
}

(Received 10 May 2016; accepted 8 September 2016; published online 18 October 2016)

Large droplets and puddles jump spontaneously from sufficiently hydrophobic surfaces during routine drop tower tests. The simple low-cost passive mechanism can in turn be used as an experimental device to investigate dynamic droplet phenomena for drops up to $10^{4}$ times larger than their normal terrestrial counterparts. We provide and/or confirm quick and qualitative design guides for such "drop shooters" as employed in drop tower tests including relationships to predict droplet ejection durations and velocities as functions of drop volume, surface texture, surface contour, wettability pattern, and fluid properties including contact angle. The latter is determined via profile image comparisons with numerical equilibrium interface computations. Water drop volumes of $0.04-400 \mathrm{ml}$ at ejection speeds of $-0.007-0.12 \mathrm{~m} / \mathrm{s}$ are demonstrated herein. A sample application of the drop jump method is made to the classic problem of low-gravity phase change heat transfer for large impinging drops. Many other candidate problems might be identified by the reader. Published by AIP Publishing. [http://dx.doi.org/10.1063/1.4963686]

\section{NOMENCLATURE}

Bo $=$ Bond number $\equiv \rho g_{o} V_{d}^{2 / 3} / \sigma$, where $\rho$ is the density difference across the fluid interface

$g \quad=$ acceleration field strength

$g_{o} \quad=$ terrestrial gravitational acceleration, $9.8 \mathrm{~m} / \mathrm{s}^{2}$

$H=$ characteristic capillary height, $2\left(\sigma / \rho g_{o}\right)^{1 / 2}$

$L_{d} \quad=$ puddle length scale

$R_{c} \quad=$ Cassie (outer) circle radius

$R_{d} \quad=$ droplet radius, $\left(3 V_{d} / 4 \pi\right)^{1 / 3}$

$R_{p} \quad=$ puddle radius

$R_{S} \quad=$ spherical solid surface (dish) radius of curvature

$R_{w} \quad=$ Wenzel (inner) circle radius

$\mathscr{R}=$ solid substrate curvature parameter, $1-R_{d} / R_{S}$

$t=$ time

$t_{d t} \quad=$ low-g freefall time duration of drop tower

$t_{j} \quad=$ drop jump time scale, $\left(\rho H R_{p}^{2} / \sigma\right)^{1 / 2}$

$T_{p} \quad=$ hot plate surface temperature

$u \quad=$ droplet impact velocity

$U \quad=$ drop/puddle jump velocity

$\tilde{U} \quad=$ numerical or simple analytic prediction of puddle jump velocity

$\tilde{U}_{\max }=$ maximum simple analytic model prediction of puddle jump velocity

$\tilde{U}_{w / c}=$ Wenzel-Cassie puddle jump velocity

$V_{w} \quad=$ remnant of volume of original puddle volume on Wenzel surface after jump

$V_{d} \quad=$ drop/puddle volume

\footnotetext{
a)E-mail: mmw@cecs.pdx.edu
} 


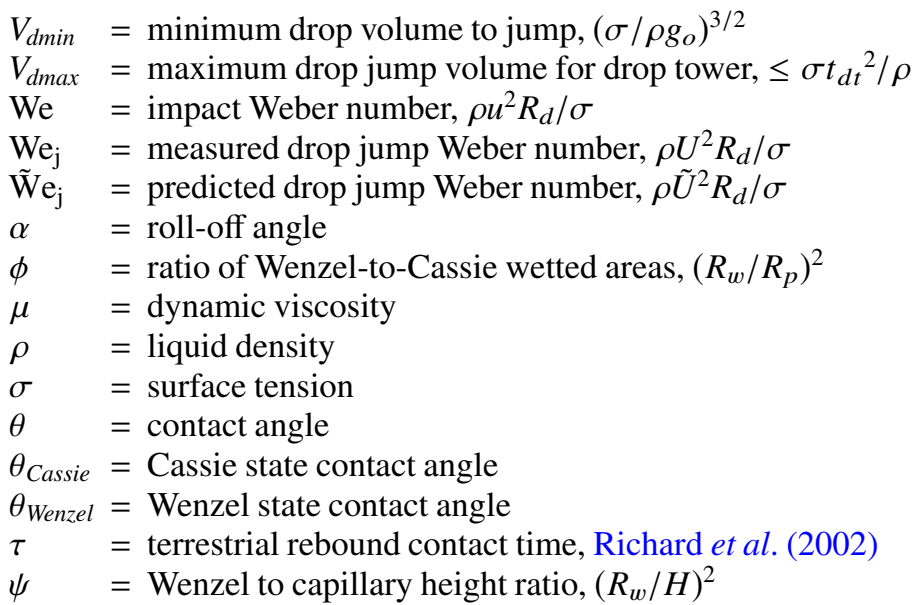

\section{INTRODUCTION}

As recently introduced and reviewed by Wollman et al. (2016), the drop jump or puddle jump mechanism provides a no-moving-parts method for investigations of large length scale drop physics. Such investigations are of fundamental interest in their own right, but they are particularly applicable to the low-g fluid systems aboard spacecraft where such large unearthly droplets are commonplace in fuel tanks, coolant systems, and water processing equipment.

A simple drop tower experiment rig is shown in Fig. 1 where a $2 \mathrm{ml}$ blue-dyed water droplet is placed on a hydrophobic surface achieving a Cassie state. To various degrees, releasing the experiment into freefall results in a "rolling-up" of the "puddle" across the surface. The vertical asymmetry of the geometry allows the drop to push off and away from the surface as shown incrementally in Fig. 2. Kirko et al. (1970) were the first to report such observations in early drop tower tests employing immiscible liquids (i.e., mercury in hydrochloric acid). A wide variety of drop-in-air demonstrations are highlighted by Weislogel (2012) and Wollman et al. (2016). The drop ejection process is related to the second half of droplet rebound and bounce phenomena from super-hydrophobic surfaces (see Richard et al. (2002) and de Ruiter et al. (2015), and references contained therein) and the drop jump phenomena observed when drops coalesce on hydrophobic surfaces (Farhangi et al., 2012). The inverse problem of bubbles jumping "downward" from flat surfaces in drop tower experiments is also discussed by Wollman et al. (2016) with related work pursued by Suñol and González-Cinca (2010). Numerical investigations of the drop jump phenomena are conducted by Zhang et al. (2014).
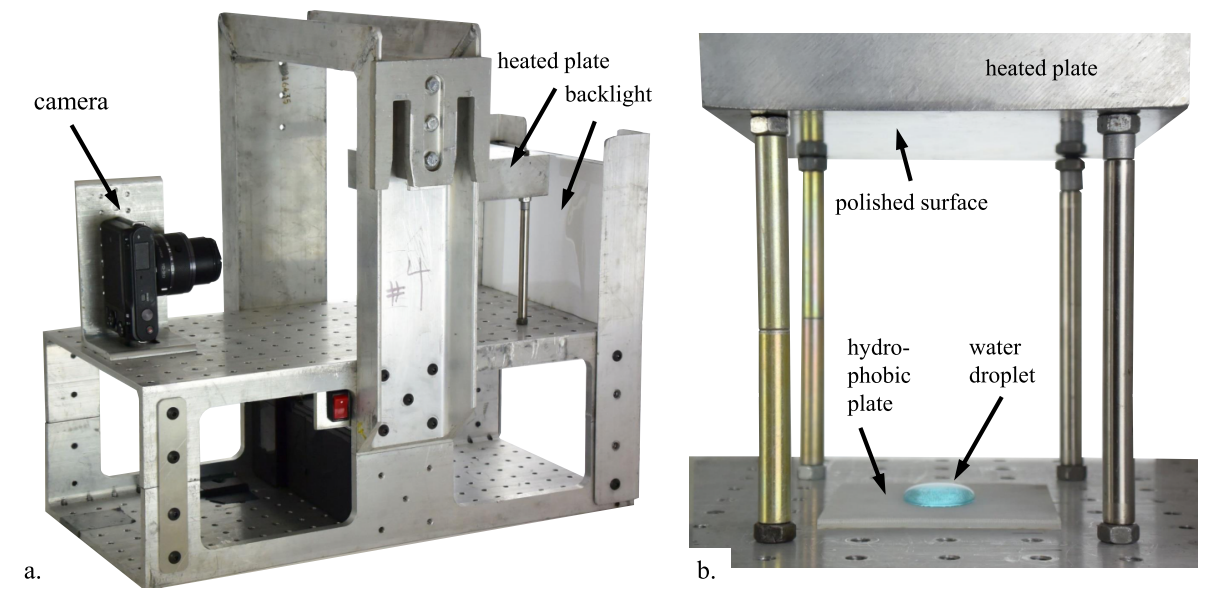

FIG. 1. (a) Simple drop tower experiment rig with (b) magnified view of drop jump surface. 


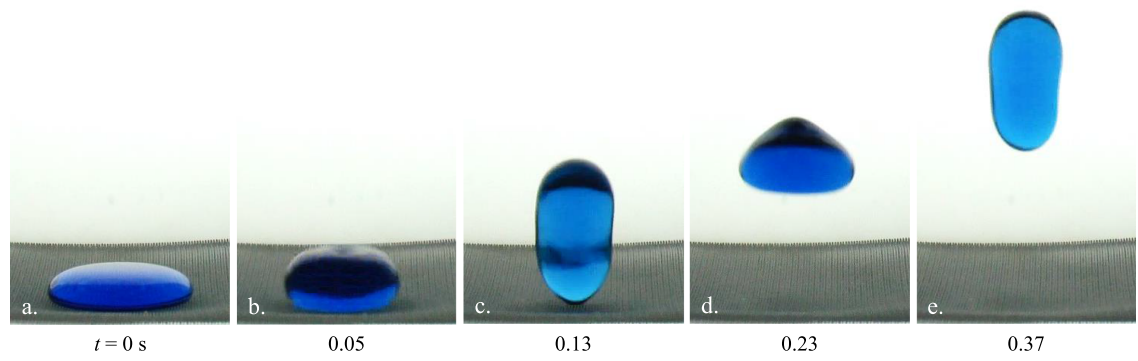

FIG. 2. Drop jump of $2 \mathrm{ml}$ water puddle from a hydrophobic surface with $\theta \approx 148^{\circ}$. (a) At $t=0 \mathrm{~s}$ the puddle is largely flat due to gravity. Following release of the experiment into freefall (b) the drop rolls up, (c) jumps from the surface at fixed velocity $U$, and (d) and (e) exhibits damped oscillations dependent on droplet volume and fluid properties.

\section{APPLICATIONS OF THE DROP JUMP MECHANISM}

Because the drop jump process is so simple, we are interested in applying it to numerous low-gravity drop dynamics investigations. For example, the experiment rig in Fig. 1 includes a polished aluminum plate positioned directly above the hydrophobic drop jump surface. This plate is heated prior to the test to any desired temperature to study large drop impacts on heated surfaces in a microgravity environment. Fig. 3 provides test results performed at interesting plate temperatures: Fig. 3(a) shows an ambient impact and damped oscillation $\left(T_{p}=25{ }^{\circ} \mathrm{C}\right), 3(\mathrm{~b})$ nucleate boiling with a majority of mass loss by bubble rupture and satellite droplet ejection $\left(T_{p}=179{ }^{\circ} \mathrm{C}\right), 3(\mathrm{c})$ transition boiling $\left(T_{p}=239{ }^{\circ} \mathrm{C}\right)$, and $3(\mathrm{~d})$ the Leidenfrost condition with miniscule heat transfer $\left(T_{p}=306{ }^{\circ} \mathrm{C}\right)$. Adhesions, splashing, bouncing, and boiling in numerous combinations are observed. A broad literature exists for this important problem (Inadat and Yang, 1993), but the drop jump mechanism via drop tower facility permits the study of drops with up to $10^{4}$ times the volume of typical terrestrial drops with accompanying large, slow, easily filmed, macro-capillary dynamics. The freefall environment also allows the study of low impact Weber numbers approaching zero impact velocity and with certifiably negligible buoyancy effects.

Without using the drop jump method, similar freefall drop impact experiments are performed, for instance, by Qiao and Chandra (1995) who employ still flash photography during $0.055 \mathrm{~s}$ "drop tests" ( $15 \mathrm{~mm}$ free fall distance) with $V_{d} \approx 0.0017 \mathrm{ml}$. The longer duration of a designated drop tower facility $(22.2 \mathrm{~m}$ and $2.1 \mathrm{~s}$ in our case) permits at least a 38 -fold longer viewing time for drops up to 200000 times larger and achieves impact Weber numbers $W e=\rho u^{2} R_{d} / \sigma$ an order of magnitude smaller, where $W e$ is a measure of the inertia present at impact relative to the restoring force of surface tension.

The breadth of experimental parameters in such investigations is large when considering surface materials and properties, fluid types, wall super-heat, liquid sub-cooling, air saturation, impact angle, oscillation mode at impact, and more. Rein (1993) discussed the importance of incident angle and droplet shape (prolate or oblate) on impact. In another study, Lee et al. (2013) examined dynamic behavior of an impinging droplet on a hot surface by varying the Weber number. Inada et al. (1989) studied mechanisms of the "miniaturization" of 6.3 and $33.5 \mu \mathrm{l}$ sessile droplets following impact with a heated plate.

In a natural way, drop volume, impact velocity, and impact oscillation mode are controlled output parameters of the drop jump method. Following this line of inquiry, the present investigation seeks to report some of the performance limits and design guides restricted to the drop jump method when employed as a drop deployment device for any number of drop dynamics investigations. Distilled water with surface tension $\sigma=71.9 \mathrm{mN} / \mathrm{m}$ and density $\rho=1000.6 \mathrm{~kg} / \mathrm{m}^{3}$ is used in all tests performed herein. Nonwetting Cassie-state contact angles between $120^{\circ} \leq \theta \leq 150^{\circ}$ are achieved. The NASA SpotLight-16 image analysis software of Klimek and Wright (2006) is used to digitize all droplet dynamics including center of mass displacements to determine drop jump velocity $U$, usually by threshold tracking. Most experiments are recorded via HD Panasonic 900M video camera at $60 \mathrm{fps}$. When needed, a Nikon J1 with $30 \mathrm{~mm}$ VR lens at $400 \mathrm{fps}$ is employed. 
a. $25^{\circ} \mathrm{C}$
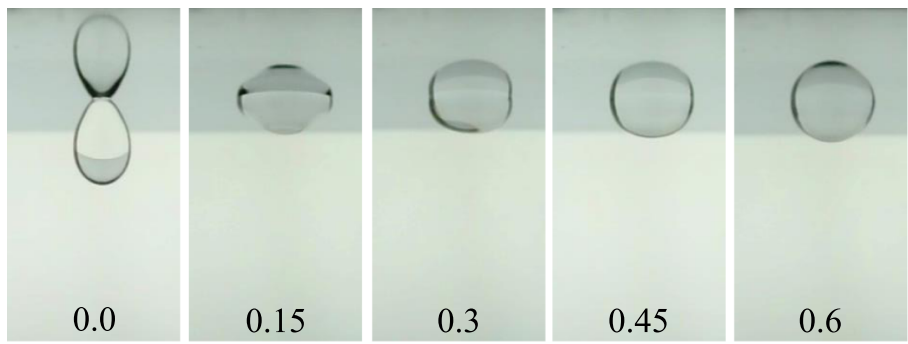

b. $179^{\circ} \mathrm{C}$
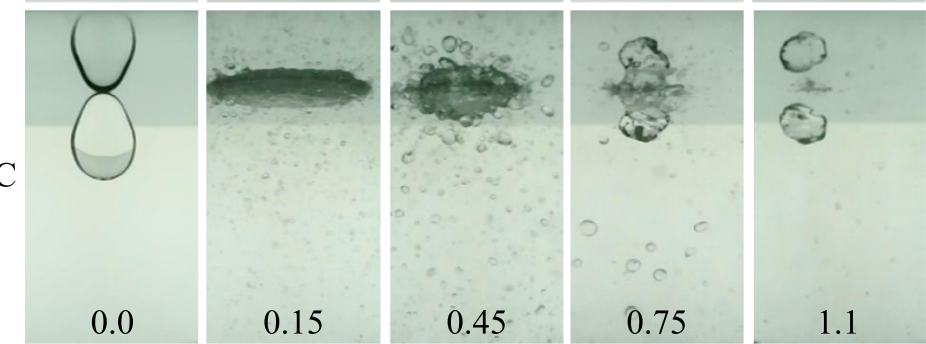

c. $239^{\circ} \mathrm{C}$
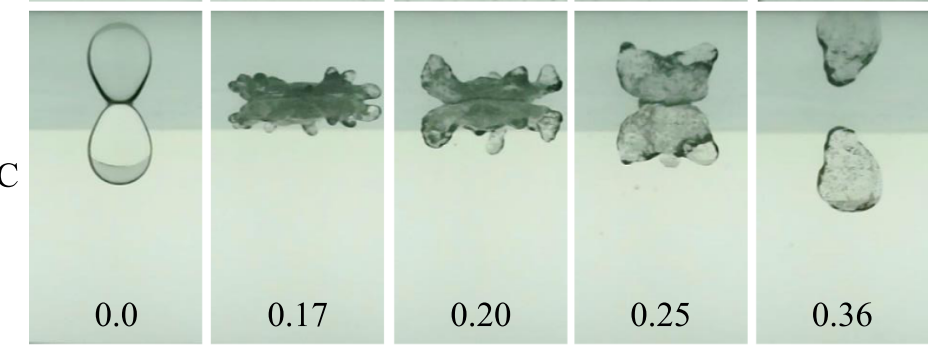

d. $306{ }^{\circ} \mathrm{C}$
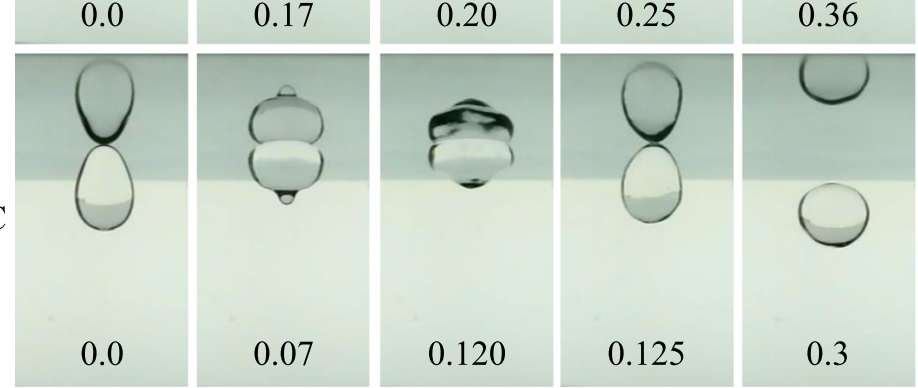

FIG. 3. A $2 \mathrm{ml}$ water drop with initial temperature $23^{\circ} \mathrm{C}$ launched via puddle jump impinges against a heated mirror polished aluminum plate: (a) $T_{p}=25{ }^{\circ} \mathrm{C}$, (b) $179{ }^{\circ} \mathrm{C}$ (nucleate boiling with significant droplet ejection), (c) $239{ }^{\circ} \mathrm{C}$, and (d) $306{ }^{\circ} \mathrm{C}$ (the Leidenfrost temperature). Times after impacts are provided in seconds. Images recorded at $400 \mathrm{fps}$.

Diffuse background lighting is provided by Green LED Lighting Solutions' 24 VDC LED backlight panels.

Up to four methods are used to measure the steady jump velocities reported herein: automated area centroid tracking, automated threshold tracking of the average of the top and bottom location of the drop, and sometimes manual tracking of the same. True mass center measurements could be made only for rare cases of axial symmetry. Between 126 and 840 images per drop jump experiment could be analyzed. For sufficient distances from the hydrophobic surfaces, the methods achieved nearly identical results with \pm 2 pixels accuracy leading to velocity errors significantly less than $\pm 5 \%$ depending on magnification. Precision mass $\pm 0.1 \%$ and precision graduated syringe $\pm 1 \%$ methods were used to determine drop volumes. The drop deployment methods were calibrated for each experimenter and found repeatable to $\pm 1 \%$. We note that slight evaporation of the deployed drops could be observed when drop mass was measured adding typically $\lesssim 1 \%$ to an over-prediction in drop volume. Over 550 drop tower tests were conducted in support of this work with individual experiments repeated as many as 10 times with nominal reproducibility of $\pm 3 \%$, which is approximately equivalent to the total individual experiment error of approximately $\pm 3 \%$. (Note that by 10 repeat experiments we mean 10 "identically" prepared surfaces, 10 "identically" deployed drops of 
"identical" volume, and 10 "identical" image processing procedures to find the nominal experiment repeatability of $U \pm 3 \%$.) We note that errors in surface preparation such as poor hydrophobic coating coverage, incomplete coating curing, contamination, local Wenzel states, and asymmetric initial conditions produce obvious jump velocity outliers that are not presented.

\section{DROP JUMP CHARACTERISTICS AND MEASUREMENTS}

Focusing strictly on the passive drop jump mechanism arising from the step reduction in effective gravity level common to drop tower facilities, we identify the primary parameters of the system as (1) drop volume, (2) surface hydrophobicity, and (3) surface topology. The impact of gravity on the process is measured by the initial $1-g_{o}$ Bond number of the system $\mathrm{Bo}=\rho g_{o} V_{d}^{2 / 3} / \sigma$. The drop tower release method and drop rig apparatus can also play a significant role in the drop jump process by introducing and promulgating acoustic ringing in the system during freefall. Such effects are unavoidable, complex, minimized by design, and presently neglected. Thus, drop volume, surface hydrophobicity, and surface topology are treated herein as control parameters to design drop jump systems for desired drop velocities, drop volumes, drop mode shapes, and more. We first present the impact of drop volume on the experimentally measured drop jump velocity. Simple estimates are forwarded for jump time, minimum and maximum jump volume, and maximum expected jump velocity. The methods used to produce large hydrophobic surfaces are then discussed followed by a selection of observations of potentially useful drop jump characteristics arising from the unique control of hydrophobic surface topology at such large capillary length scales.

\section{DROP VOLUME, $V_{d}$}

For comparison with the $2 \mathrm{ml}$ drop jump of Fig. 2, Figs. 4 and 5 provide image sequences of 30 and $95 \mathrm{ml}$ drop jump tests, respectively. The specific wetting conditions of the surfaces will be discussed shortly. From Figs. 2, 4, and 5 it is clear that the presence of inertia increases with drop volume leading eventually to bubble ingestion, geyser formation, and highly perturbed undulating puddle jumps.

For $V_{d} \leq 20 \mathrm{ml}$, the impact of drop volume on jump velocity is shown in Fig. 6 with a selection of representative scaled images of the drops at the point of ejection provided for reference. We note that typical terrestrial droplet volumes are $\lesssim 0.1 \mathrm{ml}$. The drop volume is an easily controlled variable affecting drop jump in such drop tower tests. The drop jump velocity first increases with

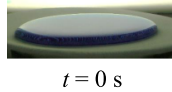

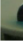

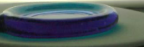

0.16

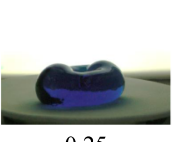

0.25

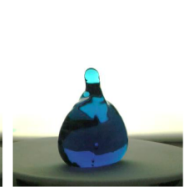

0.33

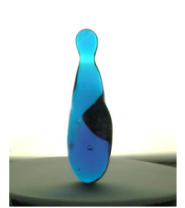

0.50

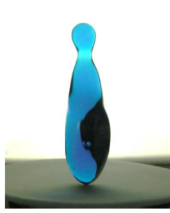

0.55

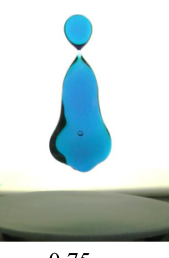

0.75

FIG. 4. A $30 \mathrm{ml}$ puddle jump.
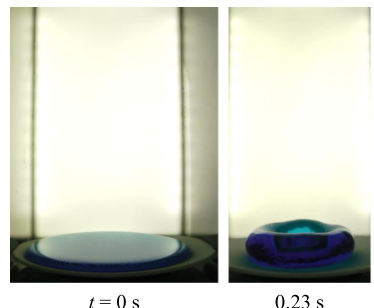

$0.23 \mathrm{~s}$

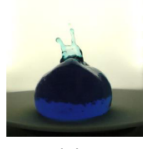

0.35

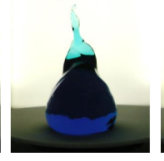

0.41

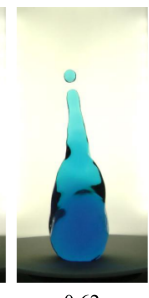

0.62

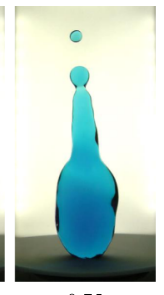

0.75

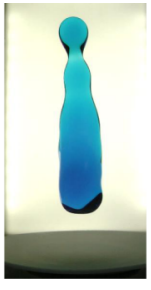

1.28

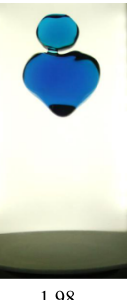

1.98

FIG. 5. Formation of a geyser at $t=0.35 \mathrm{~s}$ for a slightly asymmetric $95 \mathrm{ml}$ puddle jump. 


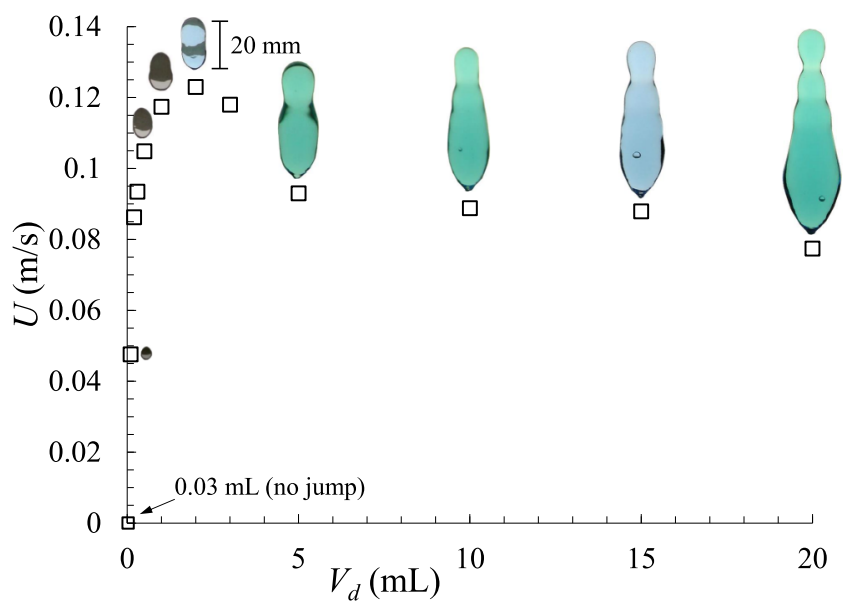

FIG. 6. Drop jump velocity $U$ as a function of drop volume $V_{d}$ (laser etched surface \#4). Select images at point of separation shown approximately to scale.

drop volume. A single frame is presented in Fig. 7 for four small drops of incremental volume tested simultaneously in this regime. As drop volume increases above $2 \mathrm{ml}$, a sharp decline in $U$ is observed in Fig. 6. A wider range of drop jump data is presented in Fig. 8 for $U$ and Fig. 9 for We. Images of the drops at the point of separation are added for comparisons in Fig. 9. The smallest image shown is for a $0.6 \mathrm{ml}$ drop which is already nearly 10 times larger than typical terrestrial drops. These results will be discussed further in connection with simple model predictions.

\section{A. Minimum drop jump volume, $V_{d m i n}$}

For sufficiently hydrophobic Cassie-state conditions, the impact of drop volume is manifest through the magnitude of Bond number, where Bo $\gg 1$ implies initially gravity-dominated, flat, disc-like puddle states that are certain to jump. Drop jump is less likely as Bo decreases in magnitude below Bo $\sim 1$ because in such instances the drop initial condition is nearly as dominated by surface tension as the low-g state of the drop tower test. Thus, we expect

$$
V_{d m i n} \sim\left(\sigma / \rho g_{o}\right)^{3 / 2},
$$

and for water, a lower volume limit of the drop jump phenomena $\approx 0.02 \mathrm{ml}$ is predicted. Such a limit for $V_{d} \approx 0.03 \mathrm{ml}$ is identified in Fig. 6 , where $\mathrm{Bo} \approx 1.32$. Lower drop volume limits are expected for higher contact angles. Nonetheless, such a threshold is expected for all wetting conditions since it is more energetically favorable for the drops to adhere to surfaces regardless of the contact angle (Satterlee and Reynolds, 1964).

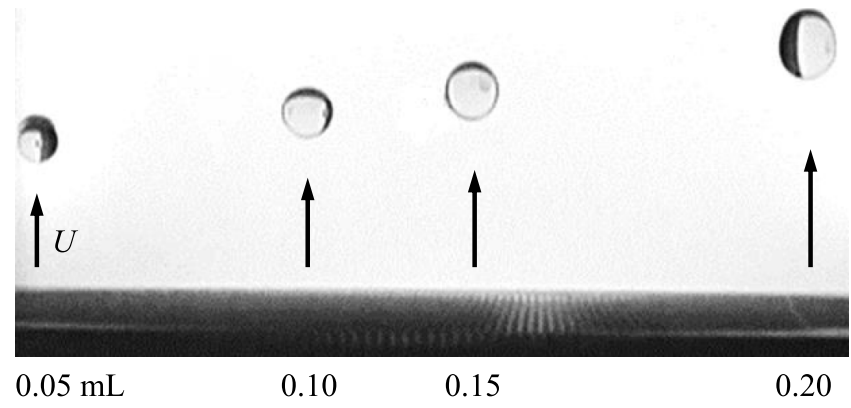

FIG. 7. Drop jump velocity $U$ resulting from drop tower test as a function of drop volume $V_{d}$ (3D printed hydrophobic surface). Image taken at $t=0.39 \mathrm{~s}$ into drop test. 


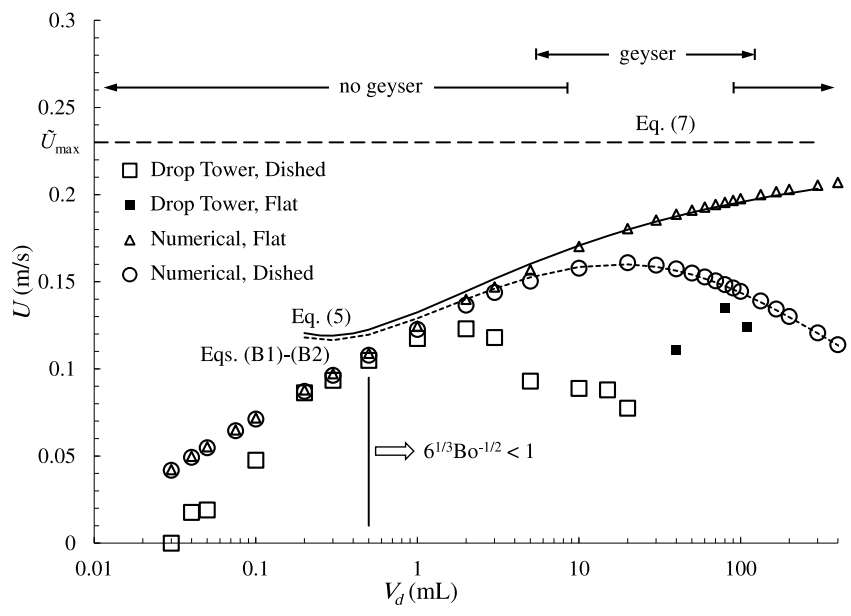

FIG. 8. Comparison of experimental drop jump velocity $U$ from a dished 3D printed hydrophobic surface $\left(R_{S}=0.352 \mathrm{~m}\right)$ with numerical and simple theoretical predictions. Relevancy for the simple theory leading to Eqs. (5), (B1) and (B2) is identified by the onset of the large puddle limit $6^{1 / 3} \mathrm{Bo}^{-1 / 2}<1 . U_{\max }$ from Eq. (7) is the asymptote for Eq. (5). Drop dimensions $(<5 \mathrm{~mm})$ begin to rival hydrophobic surface post spacing $(\approx 0.5 \mathrm{~mm})$ for $V_{d} \lesssim 0.1 \mathrm{ml}$, which is also the upper limit of terrestrial drop volumes tested by Richard et al. (2002). The conditions where geysers are observed are identified by $6 \lesssim V_{d} \lesssim 110 \mathrm{ml}$. Three solid square data points for large drops on flat laser-etched surfaces (\#4) are provided for reference.

\section{B. Drop jump transients}

Because the drop jump process requires time to develop, an upper droplet volume limit arises depending on the low-g time afforded by the particular drop tower facility. For example, for the $30 \mathrm{ml}$ puddle jump shown in Fig. 4 the large droplet manages to leave the surface during the first $0.66 \mathrm{~s}$ of the $2.1 \mathrm{~s}$ drop tower test. Knowledge of this time is helpful for drop tower experiments wishing to exploit the puddle jump method.

In a similar manner to terrestrial drop rebound experiments, during the jump period the large puddles roll up as capillary waves travel radially inward eventually colliding and forming a jet or geyser, which in turn may break up forming large and/or small satellites. For the $95 \mathrm{ml}$ puddle jump shown in Fig. 5, the initial planform shape of the puddle is not perfectly circular, which results in an asymmetric two-pronged $1.8 \mathrm{~m} / \mathrm{s}$ geyser observed at time $t=0.35 \mathrm{~s}$ and $t=0.41 \mathrm{~s}$, as observed by Pearson et al. (2013). At $60 \mathrm{fps}$ we find that for droplets up to approximately $10 \mathrm{ml}$ no geysers form,

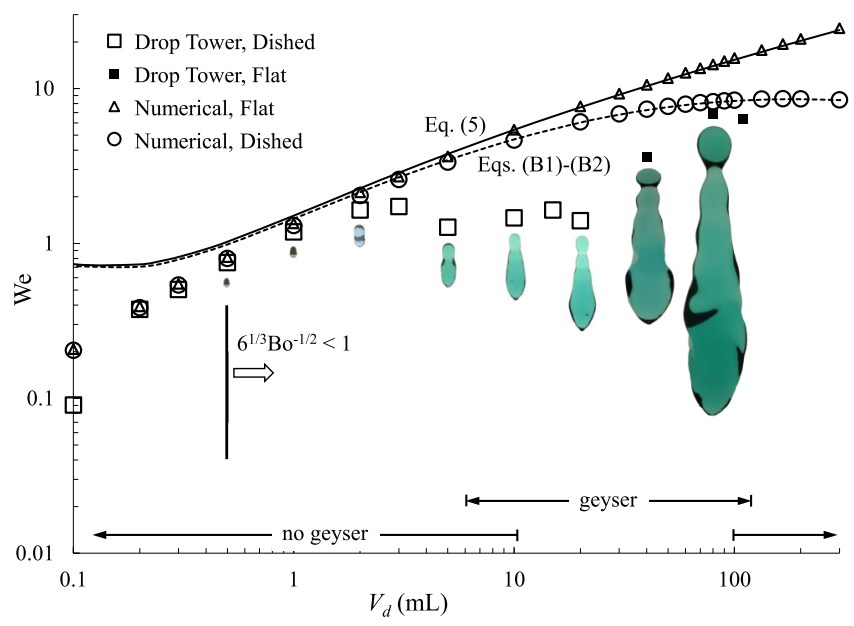

FIG. 9. Comparison of experimental jump $W_{j}$ with numerical and simple theoretical predictions. Data re-plotted from Fig. 8. Select drop jump images at separation are shown below drop tower data to approximate scale for comparisons. Satellite drops of miniscule volume in the geyser regime are not shown. 
while for drops $6 \mathrm{ml} \lesssim V_{d} \lesssim 100 \mathrm{ml}$ they do. No geysers are observed for large puddles of 200, 300, 400 , and even $500 \mathrm{ml}$. To be reported in a separate publication, subsequent experiments with higher contact angle hydrophobic surfaces suggest that geyser formation persists at higher volumes.

\section{Drop jump time scale, $t_{j}$}

The related terrestrial problem of contact time $\tau$ for a bouncing droplet impacting a hydrophobic surface is studied by Richard et al. (2002) and de Ruiter et al. (2015), and numerous others. The drop jump time of the present study is quite similar to the drop recoil and rebound phase in such investigations. In the current drop tower tests the initial condition of the large droplet is affected by gravity, but gravity does not impact the drop jump process itself. The large drops jump quickly and by ignoring viscosity $\left(\mu U R_{p} / \sigma H<<1\right)$ in addition to low-gravity $\left(\rho g R_{p} H / \sigma<<1\right)$, a scale momentum balance between inertia and surface tension gives $\rho U^{2} / R_{p} \sim \sigma / R_{p} H$, where $R_{p}$ is the $1-g_{o}$ large puddle radius defined by $\pi R_{p}^{2} H \equiv V_{d}, H \equiv 2\left(\sigma / \rho g_{o}\right)^{1 / 2}$ is the characteristic puddle capillary height, and $g$ is the acceleration level during freefall $\left(g \leq 10^{-4} g_{o}\right)$. Thus, $U \sim(\sigma / \rho H)^{1 / 2}$ and we identify the drop jump time scale as $t_{j} \sim R_{p} / U \sim\left(\rho H R_{p}^{2} / \sigma\right)^{1 / 2}$. For large disc-shaped puddles, $V_{d} \sim H R_{p}^{2}$ such that for large drops we expect the jump time scale to be

$$
t_{j} \sim\left(\frac{\rho V_{d}}{\sigma}\right)^{1 / 2},
$$

which is similar to the contact time of Richard et al. (2002) for terrestrial drop rebound experiments

$$
\tau \approx 2.6\left(\frac{\rho R_{d}^{3}}{\sigma}\right)^{1 / 2} \approx 1.27 t_{j} .
$$

Our experimentally measured drop jump values for $t_{j}$ are presented in Fig. 10 for water drops on identical laser-etched hydrophobic surfaces varying only the drop volume $V_{d}$. The data of Richard et al. (2002) with similar contact angle are also provided in the figure. Fair agreement with both Eqs. (2) and (3) is observed over the full range of drop volumes tested, which is extended by approximately 4 orders of magnitude by the present data. The contact time $\tau$ of Eq. (3) and the half-contact time $\tau / 2$ are overlaid in Fig. 10 for comparisons. Observations of the figure show that $t_{j}$ is not simply half the rebound contact time. Systematic over-predictions in jump time $t_{j}$ may be present for the largest puddles. The form of Eq. (2) is easy to remember.

\section{Maximum drop jump volume, $V_{d m a x}$}

Eq. (2) may be inverted to determine the largest characteristic puddle volume that can be expected to jump from a hydrophobic surface in the drop tower freefall time available $t_{d t}$,
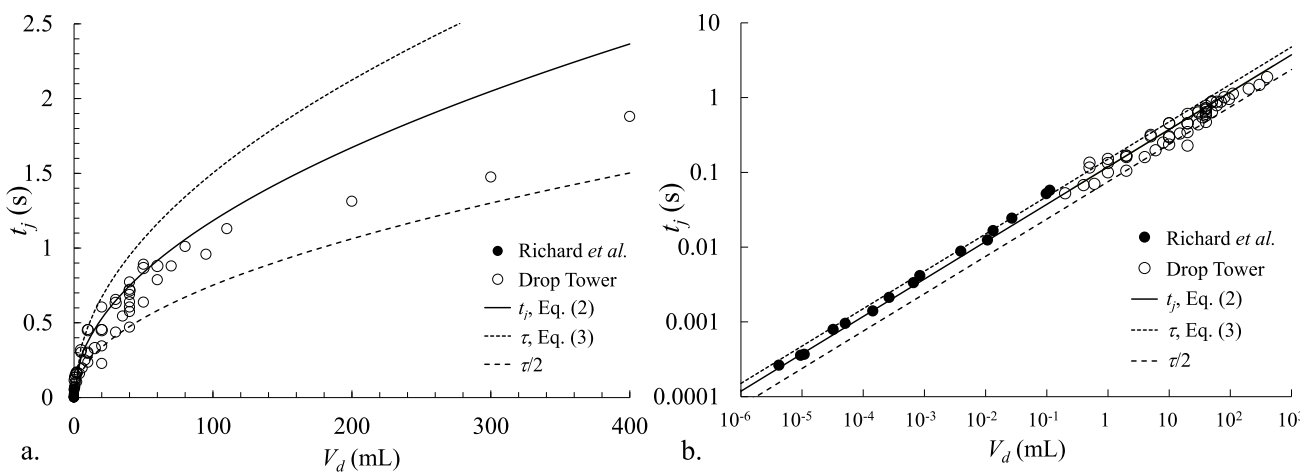

FIG. 10. Drop tower tests results (open circles) for drop jump time $t_{j}$ from laser etched surface (\#4) as function of $V_{d}$ from image data collected at $400 \mathrm{fps}$. The terrestrial measurements of drop impact and rebound by Richard et al. (2002) (solid circles) are divided by two and overlaid for comparison. A 4-order of magnitude extrapolation of the simple Eq. (2) model does well in general as seen in panel (b), with possible over-predictions beginning for $V_{d}>200 \mathrm{ml}$ as observed in panel (a). 


$$
V_{d \max } \leq \sigma t_{d t}^{2} / \rho .
$$

For example, for $t_{d t}=2.1 \mathrm{~s}$, for water, we find $V_{d \max } \approx 300 \mathrm{ml}$, which is approximately 10000 times larger than a typical raindrop $(\sim 0.03 \mathrm{ml})$. This result corroborates with experiments for our maximum drop jump volume of $400 \mathrm{ml}$ presented in Fig. 10(a).

\section{SIMPLE EQUILIBRIUM MODELS FOR PREDICTED DROP JUMP VELOCITY, $\tilde{U}$}

Simple analytic and numerical equilibrium methods may be quickly deployed to predict puddle jump velocity $\tilde{U}$ by equating the total surface energies $S E_{1}$ of the flattened drop immediately following release into freefall with the long duration spherical configuration surface energy $S E_{2}$ plus kinetic energy $K E_{2}$, the latter which is used to compute $\tilde{U}$ since $K E=\rho V_{d} \tilde{U}^{2} / 2$ and $S E_{1}=S E_{2}+K E_{2}$. All dynamic effects are ignored with expectations of over-predictions. Equilibrium contact angles, fluid properties, and initial gravity vector are imposed to compute the mechanical surface energy of the initial drop shape. The calculations may be performed for ideally flat surfaces as well as for the slightly dished surfaces employed in the drop tower experiments to keep the large puddles centered. The steady state spherical surface energy is then subtracted and drop velocity $\tilde{U}$ calculated. Only the simplest of the theoretical models is outlined below with further details for both theoretical and numerical results provided in Appendices A-C.

\section{A. Simple analytic model for drop jump velocity, $\tilde{U}$}

The drop jump velocity is estimated analytically in the large puddle volume limit by assuming a sufficiently non-wetting puddle of cylindrical disk shape with radius $R_{1}$ and height $H$. We ignore viscous losses due to shear, roll-up, receding contact line motion, and air drag. We assume the drop ultimately achieves a spherical configuration of radius $R_{2}=R_{d}$ and that no satellite drops are formed. Non-spherical distortions of the drop during the jump are eventually damped by viscous forces which are also ignored. These idealized initial and final states are depicted schematically in Figs. 11(b) and 11(c).

Using the notation of Figs. 11(b) and 11(c), the surface energies for liquid-solid $(l s)$, gas-liquid $(g l)$, and solid-gas $(s g)$ for cylindrical disk and spherical shapes are $S E_{1}=\left[(\sigma A)_{l s}+(\sigma A)_{g l}+\right.$ $\left.(\sigma A)_{s g}\right]_{1}$ and $S E_{2}=\left[(\sigma A)_{g l}+(\sigma A)_{s g}\right]_{2}$, respectively. From Young's equation, we have $\sigma_{s l}-\sigma_{s g}=$ $-\sigma_{g l} \cos \theta$, where $R_{1}=R_{p}=\left(V_{d} / \pi H\right)^{1 / 2}, R_{2}=R_{d}=\left(3 V_{d} / 4 \pi\right)^{1 / 3}$, and $H \approx 2(\sigma / \rho g)^{1 / 2}$. From $K E=S E_{1}-S E_{2}$ we find

$$
\tilde{U}=\left(\frac{\sigma g}{\rho}\right)^{1 / 4}\left[1-\cos \theta+2\left(\frac{\pi H^{3}}{V_{d}}\right)^{1 / 2}-6^{2 / 3}\left(\frac{\pi H^{3}}{V_{d}}\right)^{1 / 3}\right]^{1 / 2} \equiv\left(\frac{\sigma \tilde{\mathrm{W}} \mathrm{e}_{\mathrm{j}}}{\rho R_{d}}\right)^{1 / 2} .
$$

In the large puddle limit $\left(\pi H^{3} / V_{d}\right)^{1 / 3} \ll 1$, and for $\pi / 2 \leq \theta \leq \pi$, Eq. (5) reduces to

$$
\tilde{U} \approx\left(\frac{\sigma g}{\rho}\right)^{1 / 4}(1-\cos \theta)^{1 / 2} \text {. }
$$

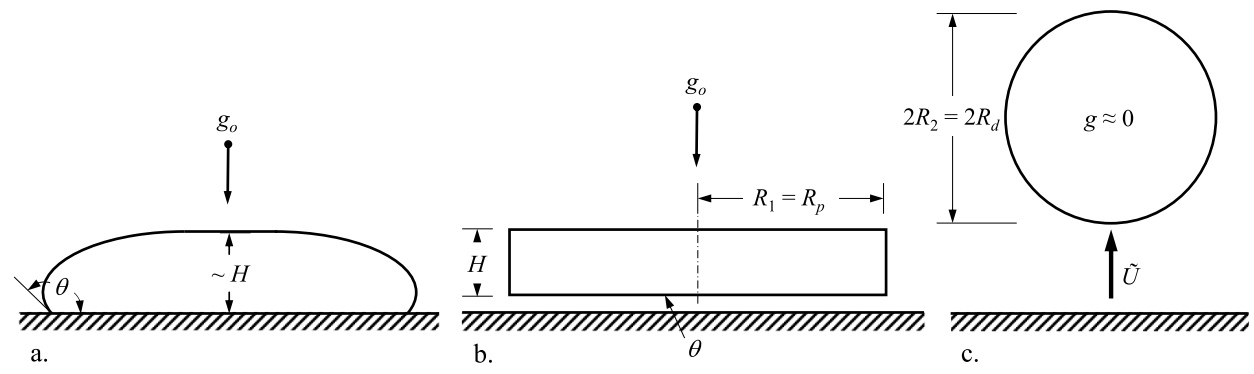

FIG. 11. Simplified large drop jump model: (a) Actual initial condition with capillary height $\sim H$, (b) state 1 idealized cylindrical disc initial condition with contact angle $\theta$ along the solid-liquid surface where $R_{1}=R_{p}$, and (c) state 2 final zero-gravity rigid body spherical configuration traveling at predicted velocity $\tilde{U}$, where $R_{2}=R_{d}$. 
When $\theta \approx \pi$ the maximum puddle jump limit is found to be

$$
\tilde{U}_{\max } \approx\left(\frac{4 \sigma g}{\rho}\right)^{1 / 4} \text {. }
$$

The dependence of $\tilde{U}$ on $\theta$ for super-hydrophobic surfaces is weak and when $\theta \approx 148^{\circ} \pm 10^{\circ}$, we observe only a $4 \%$ reduction in $\tilde{U}$ when compared to a perfect non-wetting surface of $\theta=180^{\circ}$. Substituting for $H$ we note that the large puddle limit may be recast as $\left(\pi H^{3} / V_{d}\right)^{1 / 3} \equiv 6^{1 / 3} \mathrm{Bo}^{-1 / 2} \ll$ 1 , where Bo $\equiv \rho g R_{d}{ }^{2} / \sigma$ is the Bond number based on spherical drop radius $R_{d}$. In this limit we also note that the drop jump Weber number $\tilde{\mathrm{W}} \mathrm{e}_{\mathrm{j}} \equiv \rho \tilde{U}^{2} R_{d} / \sigma \rightarrow 2 \mathrm{Bo}^{1 / 2}$. These expressions are used to write Eq. (5) as

$$
\tilde{\mathrm{W}} \mathrm{e}_{\mathrm{j}}=\mathrm{Bo}^{1 / 2}\left[1-\cos \theta+2 \cdot 6^{1 / 2} \mathrm{Bo}^{-3 / 4}-6 \mathrm{Bo}^{-1 / 2}\right] .
$$

Eqs. (5) and (7) are added to Fig. 8 providing fair agreement with experimental results, but only in the restricted regime of the analysis assumptions - above the initiation of the large puddle limit and below the limit of geyser formation (significant non-spherical distortions). Fortunately, this is a range of drop volumes that make optimal use of the brief time afforded by typical drop towers. Fig. 9 includes $\tilde{W} e_{j}$ based on predicted velocity $\tilde{U}$ from Eq. (5). In comparing terrestrial drop bounce and drop tower drop jump phenomena, since $\tilde{\mathrm{W}} \mathrm{e}_{\mathrm{j}}$ is determined by idealized equilibrium state assumptions, $\tilde{W} e_{j}$ predicts that the rebound velocity would be equal to the impact velocity. Thus, drop jump dynamics for a given value of $\tilde{\mathrm{W}} \mathrm{e}_{\mathrm{j}}$ might be expected to be similar to drop rebound dynamics when impact Weber number $\mathrm{We}=\rho u^{2} R_{d} / \sigma \approx \tilde{\mathrm{We}} \mathrm{e}_{\mathrm{j}}$.

In reference to schematics of Figs. 11(b) and 11(c), the terms in brackets in Eqs. (5) and (8) in the order left to right represent the energies of the top surface area of the disk drop, the bottom surface of the disk drop, the disk side walls, and the spherical surface, respectively. The large puddle jump velocity limit of Eq. (7) is a constant, independent of drop volume. For water we find $\tilde{U}_{\text {max }} \approx 0.23 \mathrm{~m} / \mathrm{s}$. However, $\tilde{\mathrm{We}}$ continues to rise with puddle volume through $R_{d}$, or Bo, as observed in Eq. (8) and Fig. 9. The dependence of $\tilde{U}$ on $\theta \geq 130^{\circ}$ is weak. This equilibrium analytical approach may also be pursued numerically using exact numerical surface energies for the initial surface configuration without the cylindrical disc assumption as discussed in Appendix A. Eq. (5) agrees well with the flat surface numerical solution for large drop volume.

\section{B. Other puddle jump velocity predictions, $\tilde{U}_{\text {dished }}$ and $\tilde{U}_{w / c}$}

The analytic model may be extended to address both flat and dished hydrophobic surfaces as well as drop jump phenomena for mixed Wenzel and Cassie wetting conditions. Sample calculations are provided in Appendices B and C yielding closed form expressions apt for comparisons to the experimental results in Figs. 8 and 9.

\section{SURFACE HYDROPHOBICITY}

\section{A. Hydrophobic surfaces}

Sufficiently hydrophobic Cassie state surfaces are desirable, if not essential, for the drop jump process. For the experiments of this work, we seek to quickly create large low-cost hydrophobic surfaces for a variety of drop jump investigations. Though an ever-increasing number of methods to create hydrophobic surfaces abound, such as DRIE (Sun et al., 2010), hydrophobic metals (Vorobyev and Guo, 2015), hydrophobic aerosols (Ogihara et al., 2012), and numerous commercially available coatings, in this work we construct hydrophobic surfaces by either laser etching acrylic sheets using a $60 \mathrm{~W} \mathrm{CO} \mathrm{C}_{2}$ Universal Laser Systems VLS4.60, or by 3D printing with a $30 \mu \mathrm{m}$ resolution 3D Systems Projet 3500. The requirement of surface feature control at the micro-scale is relaxed due to the large dimensions of the liquid drops under investigation here. The laser cut surfaces are created by choosing power setting $q$ and cutter speed $c$, pillar width $w$, and pillar spacing $d$ identified in Table I. For the surfaces of this work, we choose $q=36 \mathrm{~W}$ and $c=0.1 \mathrm{~m} / \mathrm{s}$. The combination of these parameters determines the channel depth $h$. The polymer surfaces are then cleaned using a warm soap solution, 
TABLE I. Properties and dimensions of hydrophobic surfaces shown in Fig. 12. Uncertainties on $\alpha$ are $\pm 10 \%$. Representative values for drop jump velocity $U$ listed for $V_{d}=2.0 \mathrm{ml}$ drop.

\begin{tabular}{lccccccc}
\hline \hline $\begin{array}{l}\text { Surface } \\
\#\end{array}$ & $\begin{array}{c}\text { Manufacture } \\
\text { technique }\end{array}$ & $\begin{array}{c}d \\
(\mathrm{~mm})\end{array}$ & $\begin{array}{c}h \\
(\mathrm{~mm})\end{array}$ & $\begin{array}{c}w \\
(\mathrm{~mm})\end{array}$ & $\begin{array}{c}\theta \\
\left({ }^{\circ}\right)\end{array}$ & $\begin{array}{c}\alpha \\
\left(^{\circ}\right)\end{array}$ & $\begin{array}{c}U \\
(\mathrm{~m} / \mathrm{s})\end{array}$ \\
\hline 1 & 3D print & 0.50 & 0.5 & 0.5 & 144 & 1.7 & 0.086 \\
2 & 3D print & 0.25 & 0.5 & 0.5 & 138 & 2.2 & 0.063 \\
3 & 3D print & 0.25 & 2.0 & 0.5 & 132 & 2.0 & 0.070 \\
4 & Laser etch & 0.50 & 0.6 & 0.4 & 148 & 2.8 & 0.120
\end{tabular}

rinsed with aqueous methanol, and air dried. They are lightly spray-coated at a $25 \mathrm{~cm}$ working distance with PTFE aerosol (King Controls, 2014) and dried with compressed air. A selection of magnified images of such surfaces is provided in Fig. 12 and characteristic properties of the surfaces are listed in Table I. Because the laser cut process is far less controlled than other methods such as photochemical etching or 3D printing, a wide variety of surface types is empirically tested using static contact angle $\theta$ and dynamic roll-off angle $\alpha$ measurements.

The example surface of Fig. 12(d) (\#4) is employed in the majority of tests reported here as it is found to establish an adequate, easily reproduced surface with contact angle $\theta \approx 148 \pm 1^{\circ}$ and roll-off angle $\alpha \approx 2.8 \pm 0.4^{\circ}$ for a $2 \mathrm{ml}$ drop. Such surfaces are sufficiently hydrophobic such that it is difficult to balance the drop on a handheld horizontal surface. As a recourse, we frequently post-process the surfaces in an oven over shallow molds (dishes) of spherical radius $R_{s}$ to assure the desired contour to accurately position the puddles before the drop tower tests.

\section{B. Measurement of contact angle $\theta$ and roll-off angle $\alpha$}

Average static non-wetting contact angles are measured by comparing profile images of drops of known volume to numerically computed droplet shapes of the same volume. The Surface Evolver algorithm of Brakke (1992) is employed via the SE-FIT graphical user interface (Chen et al., 2011). The contact angle $\theta$ is varied in the computations until the interface profile error with the experiment images is minimized. Example simulations are shown in Fig. 13 for $2 \mathrm{ml}$ and $0.03 \mathrm{ml}$ water droplets on a laser etched surface with computed contact angles $\theta \approx 148^{\circ}$ and $\theta \approx 135^{\circ}$, respectively. The numerical predictions are essentially coincident when overlain.

As often reported, droplet roll-off angles provide a convenient means of comparing the hydrophobicity of various surfaces (Smyth, 2010). For our large drops/puddles and large featured surfaces, the roll-off angle $\alpha$ is a strong function of the drop volume as shown in Fig. 14.

\section{ADDITIONAL DROP JUMP OBSERVATIONS: EFFECTS OF HYDROPHOBIC SURFACE TOPOLOGY}

\section{A. Hydrophobic surface wettability patterns}

In Fig. 15(a) is sketched a profile view of a laser etched surface where an inner circular region establishes an effective contact angle that is lower than the non-wetting area outside the circle.
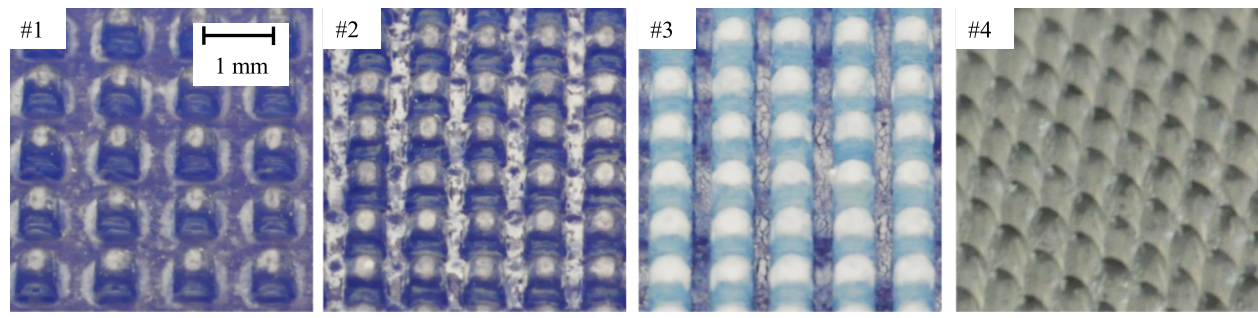

FIG. 12. 3D printed ((\#1)-(\#3)) and laser etched (\#4) surfaces employed in majority of experiments conducted. Each surface is identified by reference number with properties listed in Table I. 
a.
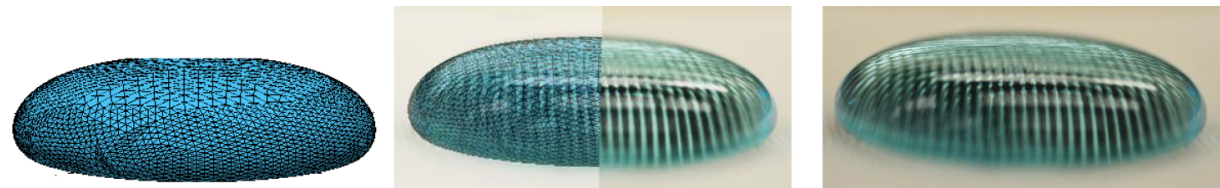

b.
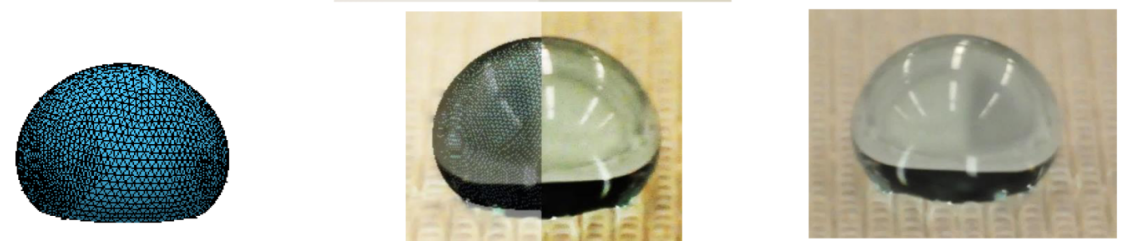

FIG. 13. (Left to right) SE-FIT simulations, overlays, and experimental images for (a) a $2 \mathrm{ml}$ puddle on a hydrophobic laser cut surface (ref. Surface \#4) with $\theta \approx 148^{\circ}$ and (b) a $0.03 \mathrm{ml}$ droplet on the same surface where $\theta \approx 135^{\circ}$.

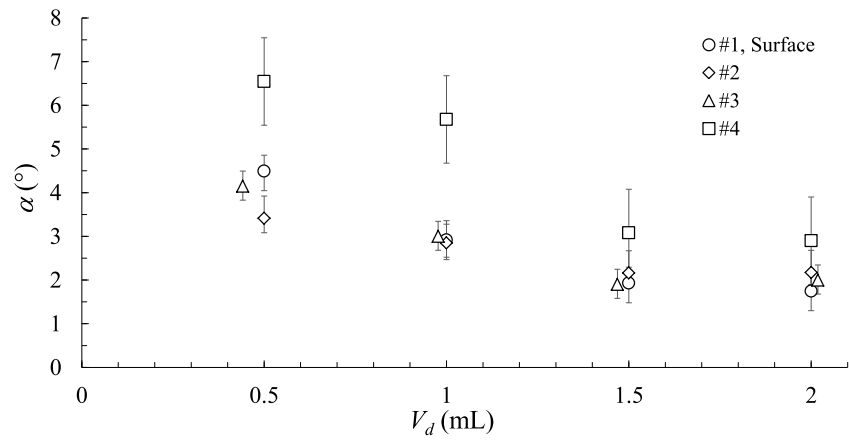

FIG. 14. Roll-off angle $\alpha$ as function of drop volume $V_{d}$ and surface type (ref. Fig. 12, Table I).
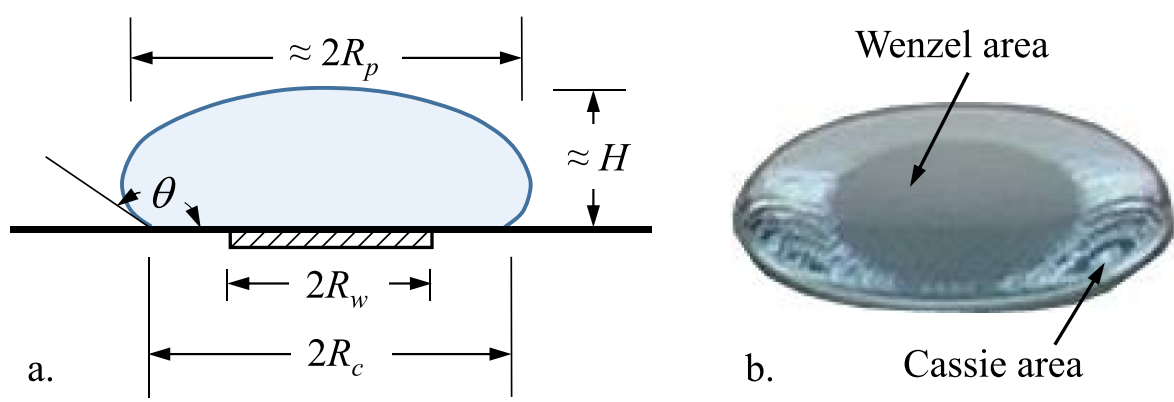

b.

Cassie area

FIG. 15. Laser etched acrylic sheet with hydrophobic surface (Cassie state) of diameter $2 R_{c}$ and partial hydrophilic surface (Wenzel state) of diameter $2 R_{w}$ : (a) schematic and (b) perspective image of a $2 \mathrm{ml}$ puddle. By varying $\phi \equiv\left(R_{w} / R_{p}\right)^{2}$ we control the drop jump velocity.

Fig. 15(b) shows a perspective image of a $2 \mathrm{ml}$ blue-dyed water drop on such a surface. By placing a drop centered on this circle with a volume that spans the wetting discontinuity, it is possible to passively tune both the volume and velocity of the jumping drop. Here we present results only when Wenzel states are established within the inner circle of radius $R_{w}$ and Cassie states within the outer circle of radius $R_{c}$. Fig. 16 presents a time sequence of the drop jump process under these conditions for $2 \mathrm{ml}$ drops with $R_{w}=3.15 \mathrm{~mm}$ and $R_{w}=4.5 \mathrm{~mm}$, respectively. We define $R_{p}$ by the large puddle limit, where $\pi R_{p}{ }^{2} H=V_{d}$ such that, for large puddles, $R_{p}$ is always slightly greater than $R_{c}$. Expectedly, the larger the area ratio $\phi \equiv\left(R_{w} / R_{p}\right)^{2}$ of Wenzel-to-Cassie wetted areas the lower the jump velocity. Fig. 17(a) presents drop jump velocity as a nearly linearly decreasing function of $\phi$. A remnant of the original puddle of volume $V_{w}$ remains on the Wenzel surface, the approximate volumes of which are plotted in Fig. 17(b). It is the degree of connection of the jumping drop with this adhered volume that controls the jump velocity. For values $\phi>0.36$, no jump occurs. For values $\phi \approx 0.306$ and 0.322 , the filament connecting the jumping drop to the adhered remnant can 

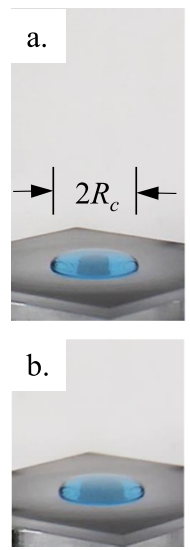

$t=0 \mathrm{~s}$
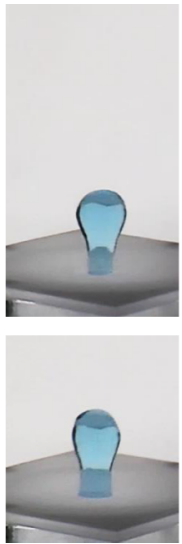

0.16
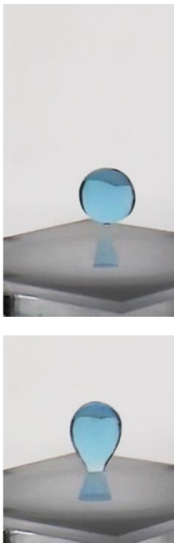

0.23
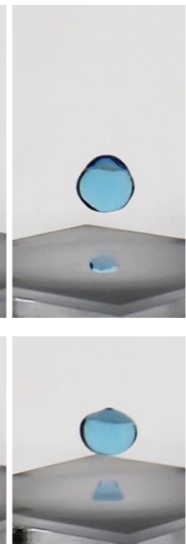

0.33
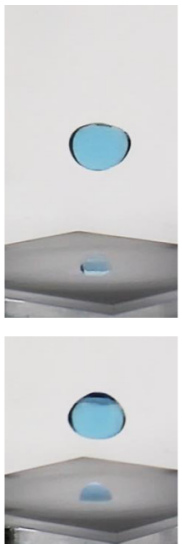

0.66
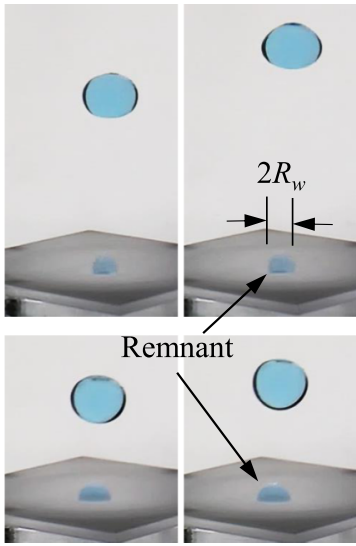

1.0
1.33

FIG. 16. A $2 \mathrm{ml}$ drop jump from a hydrophobic surface with a Wenzel state area at the center: (a) $R_{p}=11.2, R_{w}=3.1 \mathrm{~mm}$ $(\phi=0.077)$ and (b) $R_{p}=11.0, R_{w}=4.5 \mathrm{~mm}(\phi=0.17)$. The larger the Wenzel state radius $R_{w}$ the slower the jump velocity. Remnant volumes $V_{w}$ are 0.22 and $0.26 \mathrm{ml}$, respectively. For the remnant we observe $\theta_{w} \approx 115 \pm 5^{\circ}$.
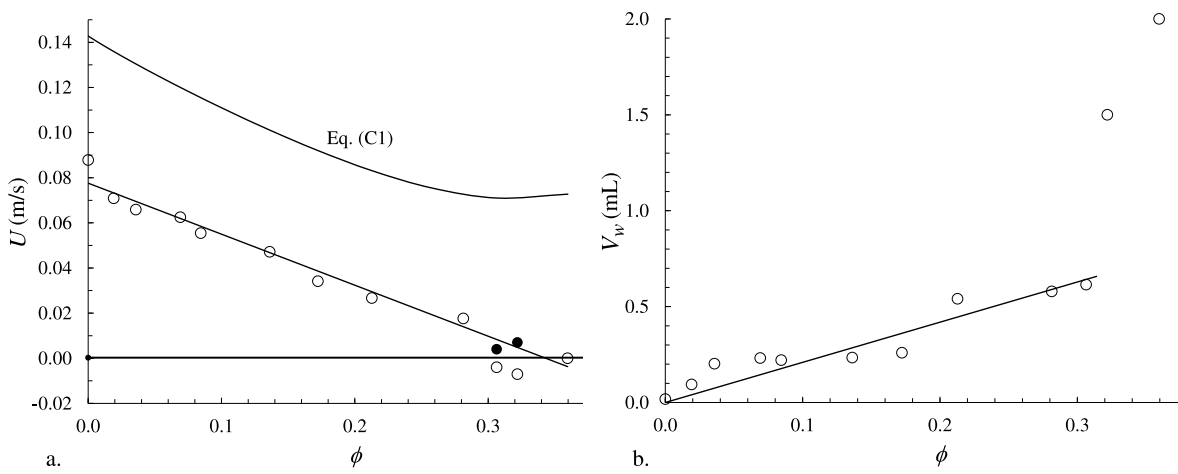

FIG. 17. (a) Drop jump velocity for $2 \mathrm{ml}$ drops as a function of Wenzel state coverage ratio $\phi$, approximately fit by $U=0.078-0.226 \phi(\mathrm{m} / \mathrm{s})$. Larger values for $\phi$ produce drops that do not jump. Open symbols represent ejected drop velocities; solid symbols represent rebounded drop velocities following collision of the ejected drop with the remnant drop. A drop of $\phi=0.36$ jumps with $U \approx 0 \mathrm{~m} / \mathrm{s}$. Larger values of $\phi$ do not jump. Eq. (C1) demonstrates qualitative agreement. (b) Wall-bound remnant drop volume as a function of $\phi$, with approximate fit $V_{w}=2.094 \phi(\mathrm{ml})$ in the linear range $\phi<0.32$.

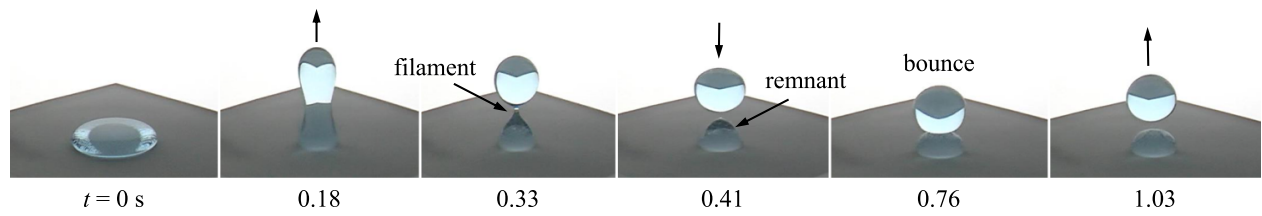

FIG. 18. A $2 \mathrm{ml}$ drop jumps from a Cassie-Wenzel surface with $\phi=0.306$. At this critical condition the retarding capillary filament force is greater than the developing ejected drop inertia $(t=0.33 \mathrm{~s})$. As the drop reverses direction towards the solid surface the rivulet ruptures $(0.41 \mathrm{~s})$, the separated drop impacts the remnant drop $(0.76 \mathrm{~s})$, rebounds, and proceeds away from the surface at a low velocity of $0.004 \mathrm{~m} / \mathrm{s}(1.03 \mathrm{~s})$. The remnant volume $V_{w}=0.62 \mathrm{ml}$ for this test.

exert a force large enough to drive the eventually detaching drop back towards the solid surface. As shown in Fig. 18, and as observed in other forced droplet ejection applications (Tanguy et al., 2012), in such cases the ejected drop bounces off the adhered remnant surface and continues its slow motion away from the surface. Negative velocities are recorded in such cases as shown in Fig. 17(a), but these vectors are reversed following rebound off the remnant drop surface. The rebound drop velocity is also reported in Fig. 17(a), which fits the overall linear trend. For these tests $\theta_{\text {Cassie }}=148^{\circ}$ and $\theta_{\text {Wenzel }} \approx 115 \pm 5^{\circ}$. Extremely low, even zero, velocity drop jumps may be 


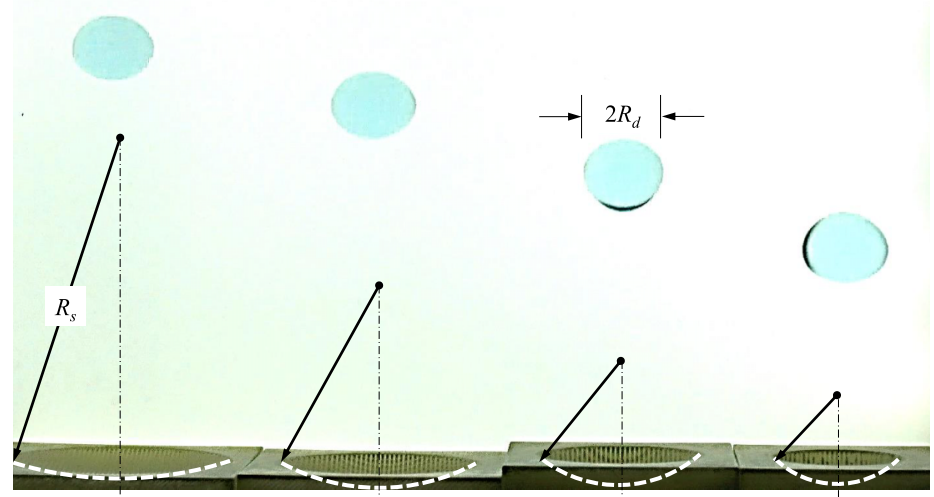

FIG. 19. Simultaneous drop jump for four $0.5 \mathrm{ml}$ water puddles from 3D printed surfaces of varying curvature at $t=0.57 \mathrm{~s}$. The curvature function $R \equiv R_{d} / R_{s}$ from left to right is $0.84,0.68,0.52$, and 0.37 , where $R_{s}=31.3,15.6,10.4$, and $7.8 \mathrm{~mm}$, respectively.

obtained in this manner, which may be of interest in subsequent investigations that hope to control the Weber number of impinging droplets to, say, heated surfaces.

\section{B. Hydrophobic surface curvature, $\mathscr{R}$}

Variations in surface topology at lengths on the order of the droplet length scale $L_{d}$ (for Bo $\gg 1, L_{d} \sim\left(\rho V_{d}^{2} g / \sigma\right)^{1 / 4}$; for $B o \sim 1, L_{d} \sim V_{d}^{1 / 3}$; for $B o \ll 1$, drops do not jump for our range on contact angles) can also be exploited to control drop jump velocity, drop oscillation mode, and vector. Fig. 19 provides a single frame of $0.5 \mathrm{ml}$ drop jumps from 4 surfaces tested simultaneously in a drop tower experiment. These 3D printed hydrophobic surfaces increment macro-curvature as sketched below each drop in the figure, where $\mathscr{R} \equiv 1-R_{d} / R_{S}$ (Moláček and Bush, 2012). Drop jump data for $0.5 \leq V_{d} \leq 2 \mathrm{ml}$ are shown for $0 \lesssim \mathscr{R} \lesssim 0.84$ in Fig. 20. The degree of reproducibility in such tests is high, typically within $\pm 3 \%$ for a $2 \mathrm{ml}$ drop with $\mathscr{R}=0.5$. We identify a nearly linear dependence of drop jump velocity on $\mathscr{R}$ as shown in Fig. 20, where $U \approx 0.103 R+0.012(\mathrm{in} \mathrm{m} / \mathrm{s})$ provides an adequate estimate for design purposes.

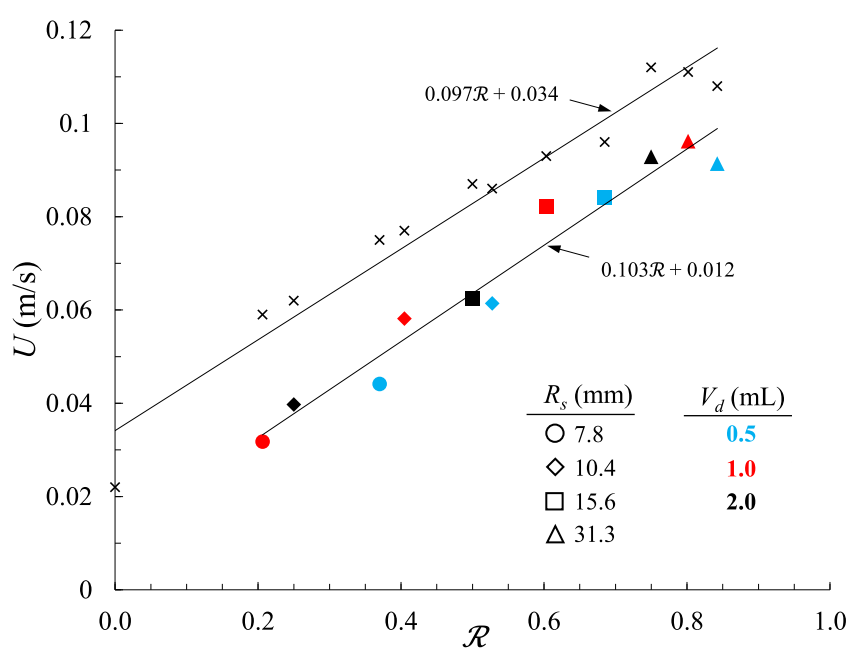

FIG. 20. Drop jump velocity $U$ as a function of 3-D printed hydrophobic surface curvature function $\mathscr{R} \equiv 1-R_{d} / R_{S}$ for selected drop volumes with $\theta \approx 145 \pm 10^{\circ}$ and hydrophobic surface spherical radius of curvature $R_{s}$. A linear relationship for $U(\mathscr{R})$ provides fair agreement for drop volumes $0.5 \leq V_{d} \leq 2.0 \mathrm{ml}$ for $\mathscr{R} \leq 0.85$. Predictions of $U$ from Eq. (B2) are provided for each test point with an " $\mathrm{x}$ " symbol. The linearly fit slopes are in favorable agreement. 


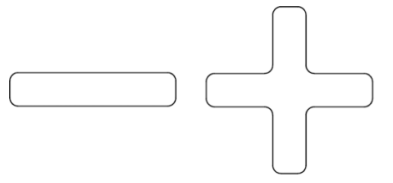

a. b.

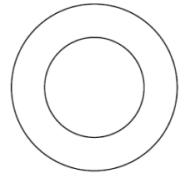

c.

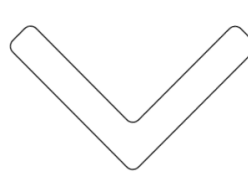

d.

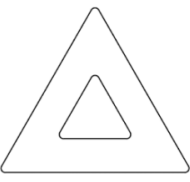

e.

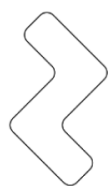

f.

FIG. 21. Depression patterns in hydrophobic surfaces tested providing a variety of jump characteristics: oblique jumps, spinning jumps, fissions, re-combinations, impacts with rebounds, and multi-mode oscillations.

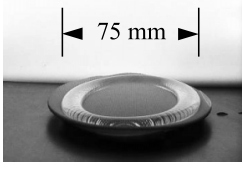

$t=0 \mathrm{~s}$

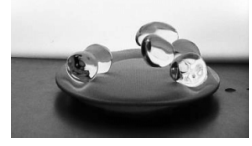

0.16

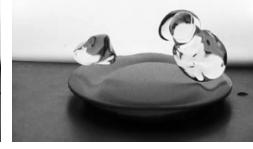

0.50

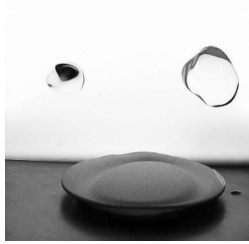

1.16

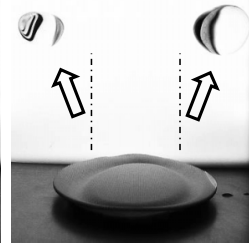

1.83

FIG. 22. Image sequence for a $10 \mathrm{ml}$ puddle jump from a hydrophobic circular trough (ref. Fig. 21(c)). In this case, the toroidal rivulet jumps, splits, and sends two approximately equal volume drops away from the surface at $\pm 8^{\circ}$ oblique angles.

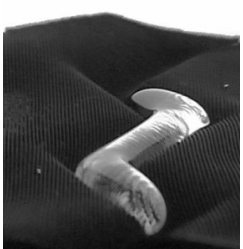

$t=0 \mathrm{~s}$

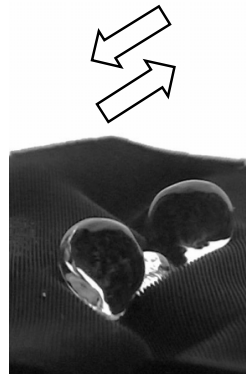

0.18

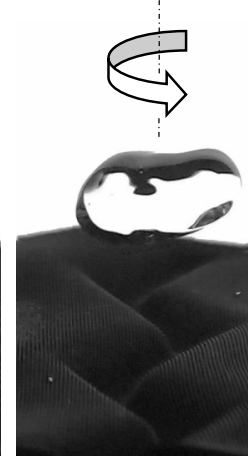

0.81

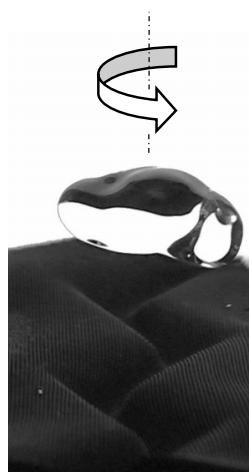

0.88

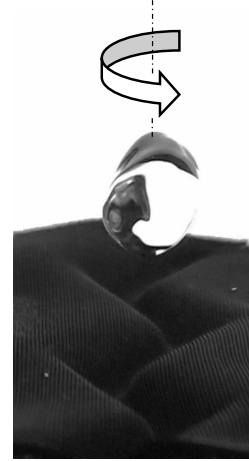

0.95

FIG. 23. Image sequence for a $9 \mathrm{ml}$ puddle jump from a hydrophobic sawtooth trough (ref. Fig. 21(f)). The sawtooth segment jumps with significant inward off-mass-center velocity components that produce a spinning drop jump with $U=0.05 \mathrm{~m} / \mathrm{s}$.

\section{Hydrophobic surface topology: Initial puddle shape}

By subsequently heating and deforming the laser etched sheets over molds, it is possible to create a variety of initial puddle shapes providing further control of drop jump characteristics such as oblique jump vectors, spin, and oscillation modes. A sub-set of patterns tested is shown in Fig. 21, the results of which only three are briefly presented herein. For example, oblique jumps are displayed in Fig. 22 where a $10 \mathrm{ml}$ circular loop puddle/rivulet (ref. Fig. 21(c)) jumps sending two drops at $\pm 8^{\circ}$ from normal angles in opposing directions. In Fig. 23, a $9 \mathrm{ml}$ rivulet in a sawtooth segment trough (ref. Fig. 21(f)) spins as it jumps. The ends of the rivulet are drawn inwards colliding off-center. The drop jump velocity for this spinning drop is $U=0.05 \mathrm{~m} / \mathrm{s}$ compared to a $9 \mathrm{ml}$ drop jump from a flat hydrophobic surface which achieves $U \approx 0.090 \mathrm{~m} / \mathrm{s}$. In Fig. 24, a $4 \mathrm{ml}$ straight line segment rivulet (ref. Fig. 21(a)) jumps at $U=0.04 \mathrm{~m} / \mathrm{s}$ with a distinctly different modal oscillation of $\approx 4.0 \mathrm{~Hz}$ when compared to normal $4 \mathrm{ml}$ circular jump with $U=0.10 \mathrm{~m} / \mathrm{s}$ and $\approx 2.5 \mathrm{~Hz}$ oscillation. Thus, by controlling the initial configuration of the drop/puddle/rivulet via hydrophobic surface topology, myriad complex interactions, oblique drops, spinning drops, and drops of varied oscillation modes may be ejected using the passive drop jump approach. 


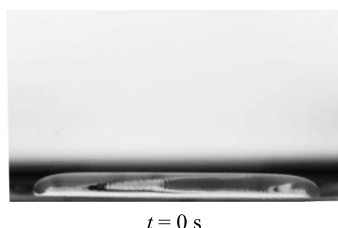

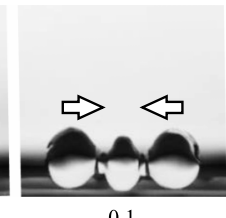

0.1

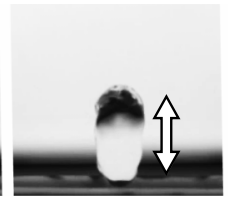

0.18

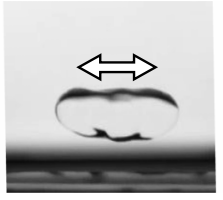

0.25

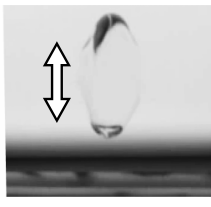

0.31

FIG. 24. Image sequence of a $4 \mathrm{ml}$ rivulet puddle jump out of a hydrophobic straight segment trough (ref. Fig. 21(a)). The cylindrical drop shapes at $t=0.33,0.47$, and $0.60 \mathrm{~s}$ are essentially identical only rotated $90^{\circ}$. The $\approx 4 \mathrm{~Hz}$ non-axisymmetric mode oscillation compares to $\approx 2.5 \mathrm{~Hz}$ axisymmetric mode for a $4 \mathrm{ml}$ planar circular puddle jump as expected.

\section{DISCUSSION}

Discrepancies of all drop jump velocity predictions with the experimental data in Figs. 8 and 9 are due primarily to the neglect of free surface distortions and viscous dissipation during and following drop jump. Best agreement is observed only for $0.5 \lesssim V_{d} \lesssim 2 \mathrm{ml}$ with significant discrepancies outside this range. Viscous dissipation due to the receding contact line, roll-up, and shear has been investigated for the drop impact and recoil problem by Attané et al. (2007) and others. For the drop jump problem, when viscous shear dominates dissipation, it may be argued that to neglect viscous dissipation the quantity $\left(\mu^{2} / \rho^{2} g_{o} V_{d}\right)^{1 / 2}$ must be exceedingly small. This condition is readily achieved for the large water drops of this investigation. For large puddle volumes $2 \mathrm{ml} \lesssim V_{d}$, the missing energy in the system must therefore reside in the large distortions of the free surface at the point of separation, which are subsequently damped by viscosity during flight. As an example, in Fig. 9, the distorted surface of the $2 \mathrm{ml}$ drop shown at separation increases the surface area of the jumped drop, which in turn reduces the energy available for bulk kinetic motion. By image analysis we estimate an approximately $7 \%$ over-prediction in kinetic energy (i.e., $\mathrm{We}_{\mathrm{j}}$ ) for this case, which is comparable to the $12 \%$ over-prediction observed in the figure. Higher harmonics may account for the remainder, but significant further work along these lines is straightforward and may be pursued. As observed in Fig. 8, at lower drop volumes $V_{d} \lesssim 0.15 \mathrm{ml}$, discrepancies of the experimental data with the numerical models are currently attributed to stick slip dissipation at the receding contact line where the characteristic surface roughness length scale $\approx 0.5 \mathrm{~mm}$ (pillar spacing) is significant compared to the characteristic drop radius $R_{d} \lesssim 3.3 \mathrm{~mm}$.

Figures 8 and 9 display the drop tower data for drop jump velocities from primarily a single dished 3D-printed hydrophobic surface with $R_{s}=0.352 \mathrm{~m}$. Flat laser-etched \#4 surface data (solid square symbols) are provided only for the largest drop volumes to indicate empirical differences expected due to surface curvature as well as to illustrate visually how large these low-gravity "droplets" can be relative to terrestrially tested droplets. Flat surface tests are increasingly difficult as drop volumes increase due to the degree of level required prior to each drop tower test. Large drops require slightly dished surfaces to prevent the drops from rolling away. However, alternative methods to establish symmetric circular puddles on flat hydrophobic surfaces may be pursued enabling comparable plots to Figs. 8 and 9 for flat surfaces and volumes up to approximately $400 \mathrm{ml}$.

\section{SUMMARY}

The drop jump method exploiting a drop tower provides a simple no-moving-parts deployment method for large droplet dynamics investigations. For sufficiently hydrophobic Cassie states $\left(\theta \geq 130^{\circ}\right)$, we show drop volume limits $0.04 \lesssim V_{d} \lesssim 400 \mathrm{ml}$ and drop velocity limits $-0.007 \lesssim$ $U \lesssim 0.12 \mathrm{~m} / \mathrm{s}$ for water in a $2.1 \mathrm{~s}$ drop tower. Zero velocity ejections may be achieved. We show adequate predictions of minimum drop jump volume $V_{d \min } \sim\left(\sigma / \rho g_{o}\right)^{3 / 2} \approx 0.02 \mathrm{ml}$, Eq. (1), maximum drop volume $V_{d \max } \sim \sigma t_{d t}{ }^{2} / \rho \approx 300 \mathrm{ml}$, Eq. (4), and certain large drop jump velocity limits $U \leq\left(4 \sigma g_{o} / \rho\right)^{1 / 4} \approx 0.23 \mathrm{~m} / \mathrm{s}$, Eq. (7). Experimental drop jump velocities are lower due to increasing dissipation during the roll-up, geysers, drop break-up, and free drop fundamental and higher harmonic capillary wave regimes. Geysers are observed for a/symmetric puddles of volumes $6 \lesssim V_{d} \lesssim 110 \mathrm{ml}$. For system design purposes, for drop volumes $0.04 \leq V_{d} \leq 400 \mathrm{ml}$ we find 
the time for drop jump $t_{j}$ adequately obeys the scale relationship $t_{j} \approx\left(\rho V_{d} / \sigma\right)^{1 / 2}$, Eq. (2). For $0.5 \lesssim V_{d} \lesssim 2 \mathrm{ml}$ the simple equilibrium model over-predicts $U$ by $\lesssim 15 \%$, Eq. (3), while a numerical equilibrium model that takes greater account of local initial interface curvature over-predicts $U$ by $\lesssim 10 \%$, Eq. (B2).

In general, the jumped droplets/puddles are enormous when compared to normal terrestrial droplet investigations; over 50-fold larger when comparing drop diameter, and over 100 000-fold larger comparing drop volume. For such uniquely large capillary fluidic bodies, the drop tower approach identifies a path for further research investigations. The drop jump velocity is not only controlled by drop volume. In this work we show that contact angle, wettability pattern, and hydrophobic surface curvature can be varied providing additional passive control of the drop jump velocity and dynamics. We find that drop jump is common for Cassie states when $\theta>130^{\circ}$ with little impact of increased contact angles in the range $150^{\circ} \lesssim \theta \leq 180^{\circ}$. By control over initial puddle shape, we demonstrate a variety of drop oscillation modes, oblique jumps, and spinning jumps. We provide simple expressions for drop jump as a function of initially combined Wenzel-Cassie states, Eq. (C1), Fig. 17, and curved hydrophobic surface, Eq. (B1), Fig. 20. We also demonstrate a fast static equilibrium numerical tool for accurate non-wetting contact angle measurement as well as a means of approximating maximum drop jump velocities while precisely determining energy losses during the process.

\section{ACKNOWLEDGMENTS}

This work was completed in part under the support of NASA Cooperative Agreement No. NNX12AO47A, the Xerox Foundation, 3D Systems, Inc., and with significant experimental support from Portland State University MCECS B.S.M.E. students L. Torres, J. Smith, K. Cardin, and J. Luce.

\section{APPENDIX A: EQUILIBRIUM NUMERICAL METHOD}

The SE-FIT software (Chen et al., 2011) which runs the Surface Evolver algorithm (Brakke, 1992 ) is used as an efficient static equilibrium numerical tool to predict puddle jump velocity $\tilde{U}$ in the manner described in Section V. Predictions of $\tilde{U}\left(V_{d}\right)$ and $\tilde{\mathrm{W}} \mathrm{e}_{\mathrm{j}}\left(V_{d}\right)$ are provided in Figs. 8 and 9 for both flat and dished hydrophobic surfaces. The numerical results are more accurate than the simple analytical results because no simplifications to the initial $1-g_{o}$ interface state and resulting surface energies are necessary. However, the numerical solution still expectedly over-predicts the experiments, the difference serving as a quick and quantitative measure of viscous dissipation and transient free surface creation during the drop jump process. Typical runtimes are less than $120 \mathrm{~s} / \mathrm{pt}$ using an Intel Xeon $2.26 \mathrm{GHz}$ 4-core desktop computer.

\section{APPENDIX B: SIMPLE ANALYTIC MODEL FOR DISHED SURFACE PUDDLE JUMP VELOCITY, $\tilde{U}_{\text {dished }}$}

The SE-FIT numerical method is applied in Figs. 8 and 9 for both flat and dished hydrophobic surfaces, as shown schematically in Figs. 11 and 25, respectively. The simple analytic model of $K E=S E_{1}-S E_{2}$ applied to the latter yields

$$
\tilde{U}_{\text {dished }}=\left[\frac{2 \sigma}{\rho V_{d}}\left(S E_{1}-S E_{2}\right)\right]^{1 / 2} \equiv\left(\frac{\sigma \tilde{W} e_{\text {dished }}}{\rho R_{d}}\right)^{1 / 2},
$$

where $S E_{1}=2 \pi h R_{S}(1-\cos \theta)-\pi h^{2}+2 \pi H\left(2 h R_{S}-h^{2}\right)^{1 / 2}$ and $S E_{2}=\left(6 \pi^{1 / 2} V_{d}\right)^{2 / 3}$ with

$$
h=R_{S}-H-2\left(R_{s}^{2}+H^{2}\right)^{1 / 2} \sin (\pi / 6-\beta)
$$

and

$$
\beta=\frac{1}{3} \tan ^{-1}\left(\frac{\left(4 \pi^{2} H^{2} R_{s}^{2}\left(3 R_{s}^{2}+2 H R_{s}+3 H^{2}\right)+12 \pi V_{d}\left(R_{s}^{3}-H^{3}\right)-9 V_{d}^{2}\right)^{1 / 2}}{2 \pi R_{s}^{3}-2 \pi H^{3}-3 V_{d}}\right) .
$$




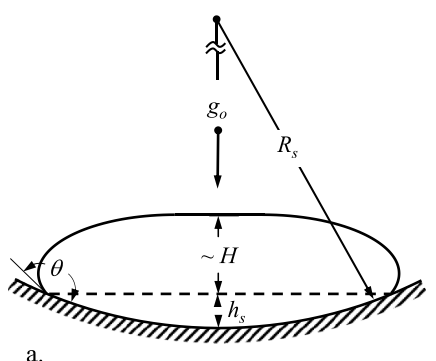

a.

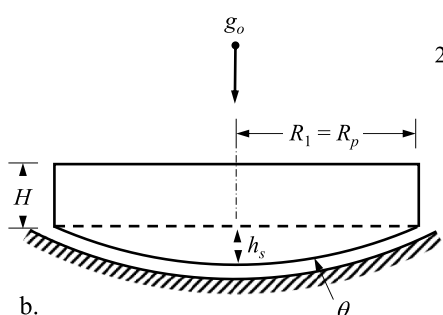

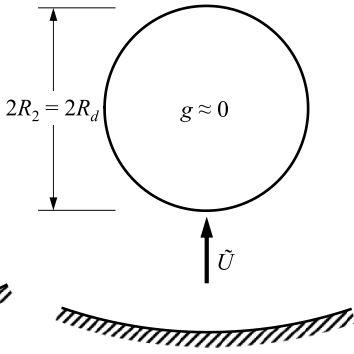

FIG. 25. Simplified large drop jump model on a spherically "dished" surface of radius $R_{s}$ : (a) Actual initial condition with capillary height $\sim H$ and dished surface depth $h_{s}$, (b) state 1 idealized cylindrical disc with spherical cap initial condition with contact angle $\theta$ along the solid-liquid surface where $R_{1}=R_{p}$, and (c) state 2 final zero-gravity rigid body spherical configuration traveling at predicted velocity $\tilde{U}$, where $R_{2}=R_{d}$.

A less cumbersome form of Eq. (B1) is found for large dish radius limit $h / R_{s} \ll 1$, which yields

$$
\tilde{U}_{\text {dished }} \approx\left[\frac{4 \pi \sigma H}{\rho V_{d} J}\left(1-\cos \theta+2 J^{1 / 2}-J\left(\frac{9}{2}\right)^{1 / 3}\left(\frac{V_{d}}{\pi H^{3}}\right)^{1 / 3}\right)\right]^{1 / 2} \equiv\left(\frac{\sigma \tilde{W} e_{\text {dished }}}{\rho R_{d}}\right)^{1 / 2},
$$

where $J \equiv H /\left[R_{s}\left(\left(1+V_{d} / \pi H^{2} R_{S}\right)^{1 / 2}-1\right)\right]$. In reference to Figs. 25(b) and 25(c), in the order left to right, the terms in the parentheses of Eq. (B2) represent the energies of the top surface area of the disk drop, the weak spherical cap bottom surface of the drop, the disk side walls, and the jumped spherical drop surface, respectively. As observed in Figs. 8 and 9, Eqs. (B1) and (B2) using the experimental value $R_{s}=0.352 \mathrm{~m}$ agree well with the equilibrium numerical solutions for large drop volume. We note also that Eqs. (B1) and (B2) recover the flat surface result of Eq. (5) in the large puddle limit $\left(\pi H^{3} / V_{d}\right)^{1 / 3} \ll 1$ when hydrophobic surface curvature is small, $V_{d} / \pi H^{2} R_{S} \ll 1$. Unlike Eq. (B2), Eq. (B1) is not limited to slightly dished hydrophobic surfaces and large puddles, making it appropriate for comparisons to the highly curved surface drop jump tests such as those presented in Fig. 20. Eq. (B1) is overlaid on the plot where it identifies the correct qualitative behavior, the differences again being a quantitative measure of energy loss during the process.

\section{APPENDIX C: SIMPLE ANALYTIC MODEL FOR WENZEL-CASSIE PUDDLE JUMP VELOCITY, $\tilde{U}_{w / c}$}

As sketched in Fig. 26, if we apply this same simple puddle jump energy approach to the Wenzel-Cassie wettability patterns of Fig. 15 we find

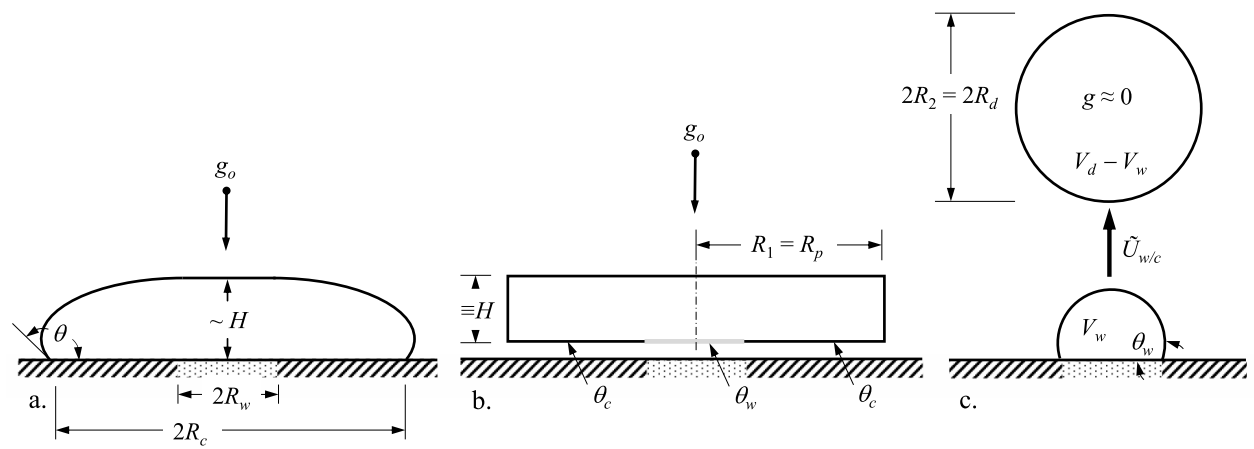

FIG. 26. Simplified large drop jump model for Cassie-Wenzel drop jump: (a) Actual initial condition with Wenzel surface identified, (b) state 1 idealized cylindrical disc initial condition, and (c) state 2 final zero-gravity rigid body spherical drop jump configuration traveling at velocity $\tilde{U}_{w / c}$, where $R_{2}=R_{d}$. The remnant drop volume $V_{w}$ resides on the Wenzel region with $\theta_{w}$. 
$\tilde{U}_{w / c}=\left(\frac{\sigma g}{\rho}\right)^{1 / 4}\left[1-(1-\phi) \cos \theta_{c}+2\left(\frac{\phi}{\psi}\right)^{1 / 2}-6^{2 / 3}\left(\frac{\phi}{\psi}\right)^{1 / 3}\left(1-\frac{f_{2} \phi \psi^{1 / 2}}{3}\right)^{2 / 3}-2 f_{1} \phi\right]^{1 / 2}$,

where $\phi \equiv\left(R_{w} / R_{p}\right)^{2}, \psi \equiv\left(R_{w} / H\right)^{2}, \theta_{c}$ is the Cassie state contact angle, $\theta_{w}$ is the effective Wenzel state contact angle, and $f_{1}$ and $f_{2}$ are dimensionless geometric functions characterizing the remnant drop free surface area and volume, respectively,

$$
f_{1} \equiv \frac{1-\cos \theta_{w}}{\sin ^{2} \theta_{w}} \quad \text { and } f_{2} \equiv \frac{\left(1-\cos \theta_{w}\right)^{3}\left(2+\cos \theta_{w}\right)}{\sin ^{3} \theta_{w}} .
$$

In reference to Figs. 15, 26(b), and 26(c), in the order left to right, the terms in brackets in Eq. (C1) represent the energies of the top surface area of the disk drop, the effective Cassie-Wenzel state bottom surface of the disk drop, the disk side walls, the reduced spherical drop surface, and the remnant drop surfaces, respectively. In the large puddle limit $\left(\pi H^{3} / V_{d}\right)^{1 / 3}=(\phi / \psi)^{1 / 3} \ll 1$, and assuming $\theta_{c} \geq 150^{\circ}$ and $\theta_{w} \approx 90^{\circ}$, Eq. (B2) reduces to

$$
\tilde{U}_{w / c} \approx\left(\frac{4 \sigma g}{\rho}\right)^{1 / 4}\left(1-\frac{\phi}{2}-\phi \cos \theta_{w}\right)^{1 / 2} \approx\left(\frac{4 \sigma g}{\rho}\right)^{1 / 4}\left(1-\frac{\phi}{4}+\cdots\right),
$$

the right hand side of which anticipates a negative linear slope form in Fig. 17(a) for small $\phi$. Eq. (C1) is added to Fig. 17(a), where it is again only helpful in a qualitative sense to predict $U$, while in a quantitative sense to predict dissipation.

Attané, P., Girard, F., and Morin, V., "An energy balance approach of the dynamics of drop impact on a surface,” Phys. Fluids 19, 012101 (2007).

Brakke, K. A., "The surface evolver," Exp. Math. 1, 141-165 (1992); the manual and code are available at http://www.susqu.edu/facstaff/b/brakke/.

Chen, Y., Schaeffer, B. M., Weislogel, M. M., and Zimmerli, G. A., "Introducing SEFIT: A surface evolver based tool for studying capillary surfaces," AIAA Paper No. 2011-1319, presented at the 49th AIAA Aerospace Sciences Meeting, 4-7 January 2011.

de Ruiter, J., Lagraauw, R., Mugele, F., and van den Ende, D., "Bouncing on thin air: how squeeze forces in the air film during non-wetting droplet bouncing lead to momentum transfer and dissipation,” J. Fluid Mech. 776, 531-567 (2015).

Farhangi, M. M., Graham, P. J., Choudhury, N. R., and Dolatabadi, A., "Induced detachment of coalescing droplets on super-hydrophobic surfaces," Langmuir 28(2), 1290-1303 (2012).

Inadat, S. and Yang, W. J., "Mechanisms of miniaturization of sessile drops on a heated surface," Int. J. Heat Mass Transfer 36(6), 1505-1515 (1993)

Inada, S., Nishida, K., Toriba, M., and Uchida, N., "Transient heat transfer for water drops impinging on heated surface for water drops impinging on heated surface,” Trans. Jap. Soc. Mech. Eng., Ser. B 55, 3499-3506 (1989).

King Controls, 2014, information about aerosol spray Dome Magic, a King Trademark, retrieved from http://www. kingcontrols.com.

Kirko, I. M., Dobychin, E. I., and Popov, V. I., "Phenomenon of the capillary bounce of a 'ball game' in weightlessness," Dok1. USSR Acad. Sci. 192, 301-303 (1970).

Klimek, R. and Wright, T., Spotlight-8 Image Analysis Software, NASA/TM-2006-214084, January 2006, available for download at https://spaceflightsystems.grc.nasa.gov/spotlight/index.html.

Lee, C. H., Kim, D. Y., Kim, H. D., and Kim, K. C., "Dynamic behavior and heat transfer characteristics of impinging droplets onto high temperature plate," presented at PIV13: The 10th International Symposium on Particle Image Velocimetry, Delft, The Netherlands, 1-3 July 2013.

Moláček, J. and Bush, J. W., "A quasi-static model of drop impact," Phys. Fluids 24(12), 127103 (2012).

Ogihara, H., Xie, J., Okagaki, J., and Saji, T., "Simple method for preparing superhydrophobic paper: Spray-deposited hydrophobic silica nanoparticle coatings exhibit high water-repellency and transparency," Langmuir 28(10), 4605-4608 (2012).

Pearson, J. T., Maynes, D., Bilodeau, D., and Webb, B. W., "Two-pronged jet formation caused by droplet impact on anisotropic superhydrophobic surfaces," in ASME Proceedings, 13th Symposium on Fundamental Issues and Perspectives in Fluid Mechanics (American Society of Mechanical Engineers, 2013), Paper No. FEDSM2013-16629, pp. V01BT16A010.

Qiao, Y. M. and Chandra, S., "Impact of n-heptane droplets on a hot surface in low gravity," Trans. Can. Soc. Mech. Eng. 19(3), 271-284 (1995).

Richard, D., Clanet, C., and Quéré, D., "Surface phenomena: Contact time of a bouncing drop," Nature 417, 811 (2002).

Rein, M., "Phenomena of liquid drop impact on solid and liquid surfaces," Fluid Dyn. Res. 12, 61-93 (1993).

Satterlee, H. M. and Reynolds, W. C., "The dynamics of the free liquid surface in cylindrical containers under strong capillary and weak gravity conditions," Technical Report No. LG-2, Department of Mechanical Engineering, Stanford University, Stanford, CA, 1964.

Smyth, K. M., "Wetting hysteresis and droplet roll off behavior on superhydrophobic surfaces," Ph.D. dissertation, Department of Mechanical Engineering, Massachusetts Institute of Technology, 2010.

Sun, G., Gao, T., Zhao, X., and Zhang, H., "Fabrication of micro/nano dual-scale structures by improved deep reactive ion etching," J. Micromech. Microeng. 20(7), 075028 (2010). 
Suñol, F. and González-Cinca, R., "Rise, bouncing and coalescence of bubbles impacting at a free surface," Colloids Surf. A 365(1), 36-42 (2010).

Tanguy, L., Liang, D., Zengerle, R., and Koltay, P., “Droplet break-up with negative momentum fluid dynamics videos,” e-print arXiv:1210.4078, October, 3 pages, fluid dynamics video (HD, LD), APS-DFD 2012, http://adsabs.harvard.edu/cgi-bin/ bib_query?arXiv:1210.4078.

Vorobyev, A. Y. and Guo, C., “Multifunctional surfaces produced by femtosecond laser pulses,” J. Appl. Phys. 117, 033103 (2015).

Weislogel, M. M., "Recent capillary fluidics research relevant to spacecraft system design," in 7th International Symposium on Two-Phase Systems for Ground and Space Applications (Chinese Academy of Sciences, Beijing, China, 2012), pp. 17-21.

Wollman, A., Weislogel, M., Wiles, B., Pettit, D., and Snyder, T., "More investigations in capillary fluidics using a drop tower," Exp. Fluids 57(4), 57 (2016).

Zhang, L., Li, Z. D., and Zhao, J. F., "Rebound of liquid droplets caused by sudden decrease of gravity," Interfacial Phenom. Heat Transfer 2(1), 41-54 (2014). 